\title{
Genome architecture marked by retrotransposons modulates predisposition to DNA methylation in cancer
}

\author{
Marcos R.H. Estécio, ${ }^{1,9}$ Juan Gallegos, ${ }^{2}$ Céline Vallot, ${ }^{3}$ Ryan J. Castoro, ${ }^{1}$ Woonbok Chung, ${ }^{1}$ \\ Shinji Maegawa, ${ }^{1}$ Yasuhiro Oki, ${ }^{4}$ Yutaka Kondo, ${ }^{5}$ Jaroslav Jelinek, ${ }^{1}$ Lanlan Shen, ${ }^{6}$ Helge Hartung, ${ }^{7}$ \\ Peter D. Aplan, ${ }^{7}$ Bogdan A. Czerniak, ${ }^{8}$ Shoudan Liang, ${ }^{2}$ and Jean-Pierre J. Issa ${ }^{1,9}$ \\ ${ }^{1}$ Department of Leukemia, The University of Texas MD Anderson Cancer Center, Houston, Texas 77030, USA; ${ }^{2}$ Department \\ of Bioinformatics and Computational Biology, The University of Texas MD Anderson Cancer Center, Houston, Texas 77030, USA; \\ ${ }^{3}$ CNRS, UMR 144, Institut Curie, 75248 Cedex 05, Paris, France; ${ }^{4}$ Department of Hematology and Cell Therapy, Aichi Cancer Center \\ Hospital and Research Institute, Nagoya 464-8681, Japan; ${ }^{5}$ Division of Molecular Oncology, Aichi Cancer Center Hospital and \\ Research Institute, Nagoya 464-8681, Japan; ${ }^{6}$ Department of Pediatrics, Baylor College of Medicine, Houston, Texas 77030, USA; \\ ${ }^{7}$ Genetic Branch, Center for Cancer Research, National Cancer Institute, National Institutes of Health, Bethesda, Maryland 20889, \\ USA; ${ }^{8}$ Department of Pathology, The University of Texas MD Anderson Cancer Center, Houston, Texas 77030, USA
}

\begin{abstract}
Epigenetic silencing plays an important role in cancer development. An attractive hypothesis is that local DNA features may participate in differential predisposition to gene hypermethylation. We found that, compared with methylationresistant genes, methylation-prone genes have a lower frequency of SINE and LINE retrotransposons near their transcription start site. In several large testing sets, this distribution was highly predictive of promoter methylation. Genomewide analysis showed that $22 \%$ of human genes were predicted to be methylation-prone in cancer; these tended to be genes that are down-regulated in cancer and that function in developmental processes. Moreover, retrotransposon distribution marks a larger fraction of methylation-prone genes compared to Polycomb group protein (PcG) marking in embryonic stem cells; indeed, PcG marking and our predictive model based on retrotransposon frequency appear to be correlated but also complementary. In summary, our data indicate that retrotransposon elements, which are widespread in our genome, are strongly associated with gene promoter DNA methylation in cancer and may in fact play a role in influencing epigenetic regulation in normal and abnormal physiological states.
\end{abstract}

[Supplemental material is available online at http:// www.genome.org.]

DNA methylation, a common cause of gene silencing in human neoplasia, does not affect the genome homogeneously: While some genes are frequently found methylated in cancer, others are never or rarely targeted by such modification. The causes of selective predisposition to methylation are unknown, and two main explanations have been proposed: (1) DNA methylation happens randomly, and cells with silencing of genes that promote tumorigenesis have a selective advantage; and (2) some genes are preferentially targeted by silencing complexes, and thus DNA methylation occurs through an instructive mechanism (Baylin and Bestor 2002; Jones and Baylin 2002; Feltus et al. 2006). The latter explanation appears more likely because genes with similar function show differential predisposition to methylation. The DNA repair genes MLH1 (frequently methylated) and MSH2 (never methylated) are good examples of genes with similar function but differential predisposition to hypermethylation in cancer (Kondo et al. 2000).

Previous studies were conducted to predict DNA methylation in individual $\mathrm{CpG}$ island promoters based on the instructive mechanism hypothesis, but despite efforts, the cause of such selective predisposition to aberrant methylation is still incompletely understood. Most commonly these studies employed support vector

\footnotetext{
${ }^{9}$ Corresponding authors.
}

E-mail mestecio@mdanderson.org.

E-mail jpissa@mdanderson.org.

Article published online before print. Article and publication date are at http://www.genome.org/cgi/doi/10.1101/gr.107318.110. machines to annotate short DNA sequences enriched in methylated versus nonmethylated gene promoter CpG islands. Although important, these studies were focused on normal cells (Bock et al. 2006; Das et al. 2006) or artificially induced methylation (Feltus et al. 2006) rather than cancer cells, which is the main system where aberrant methylation occurs. An apparent lower frequency of SINE retrotransposons in methylation-prone genes was noticed in some of these studies, including a report on hyperconserved CpG domains (Tanay et al. 2007), but this association was not further evaluated. This is an important observation with possible biological consequences, because retrotransposons such as long and short interspersed nuclear elements (LINE and SINE, respectively) are targeted by epigenetic modifications to suppress their mobilization (Yoder et al. 1997), have been shown to act as methylation centers (Yates et al. 1999), and have a strong influence on gene expression in mammalian cells (Faulkner et al. 2009). In this study, we directly investigated whether CpG island-associated genes that undergo frequent aberrant DNA methylation in cancer have a unique distribution of repetitive elements.

\section{Results}

A predictive model of predisposition to DNA methylation in cancer

To answer whether methylation-prone genes in cancer have a different distribution of repetitive elements, we first compared the 
DNA sequence in the 4 -kb region surrounding the transcription start site (TSS) of a training set composed of 36 methylation-prone and 36 methylation-resistant genes (Supplemental Table S1). Methylation analysis of the promoter region of these genes was done using quantitative methods (bisulfite-PCR followed by combined bisulfite restriction analysis [COBRA] or pyrosequencing analysis) in nine cancer cell lines. DNA from peripheral blood mononuclear cells of a healthy individual was used as a control (Supplemental Table S2). These nine cancer cell lines were selected because they were identified as heavily methylated in a previous study (Shen et al. 2007a). Thus, genes not found methylated in this group of cell lines are less likely to be found methylated elsewhere. The genes included in the training set were selected from our database of DNA methylation in cancer, and the criteria for inclusion were the following: (1) Methylation data were available for at least two cancer cell lines, (2) the gene promoter overlapped with or had a CpG island no more than 200 bp distant from the TSS, and (3) the CpG island was not methylated in the normal control. The classical Gardiner-Frommer definition of CpG islands was adopted in our study (Gardiner-Garden and Frommer 1987). As shown in Figure 1A, SINE and LINE repeats were approximately half as common in methylation-prone compared to methylation-resistant genes. Among the other repeat classes, both long terminal repeat (LTR) and DNA elements showed a lower frequency in methylation-prone genes; however, this difference was modest and not statistically significant. CpG island length, GC content, and CpG ratio were previously shown to be associated with methylation status in somatic tissues (Weber et al. 2007), but these variables were not significantly different between methylation-resistant and methylationprone genes in cancer (Fig. 1B).

To gain further information about differences in the distribution of SINE and LINE repeats between methylationprone and methylation-resistant genes, we extended the annotation to a $20-\mathrm{kb}$ region centered in the TSS in 1-kb nonoverlapping windows. The depletion of SINE repeats in methylated genes spanned nearly the full $20-\mathrm{kb}$ region, whereas the depletion of LINE repeats in methylated genes occurred mainly in a $-2-\mathrm{kb}$ to $+5-\mathrm{kb}$ window (Fig. 1C; Supplemental Fig. S1). We calculated the log-odds score of SINE and LINE retrotransposons per 1-kb window based on their distribution in the training set and in the full collection of human promoter CpG islands (Fig. 2). The sum of log-odds scores in the $20-\mathrm{kb}$ region

B.

C.
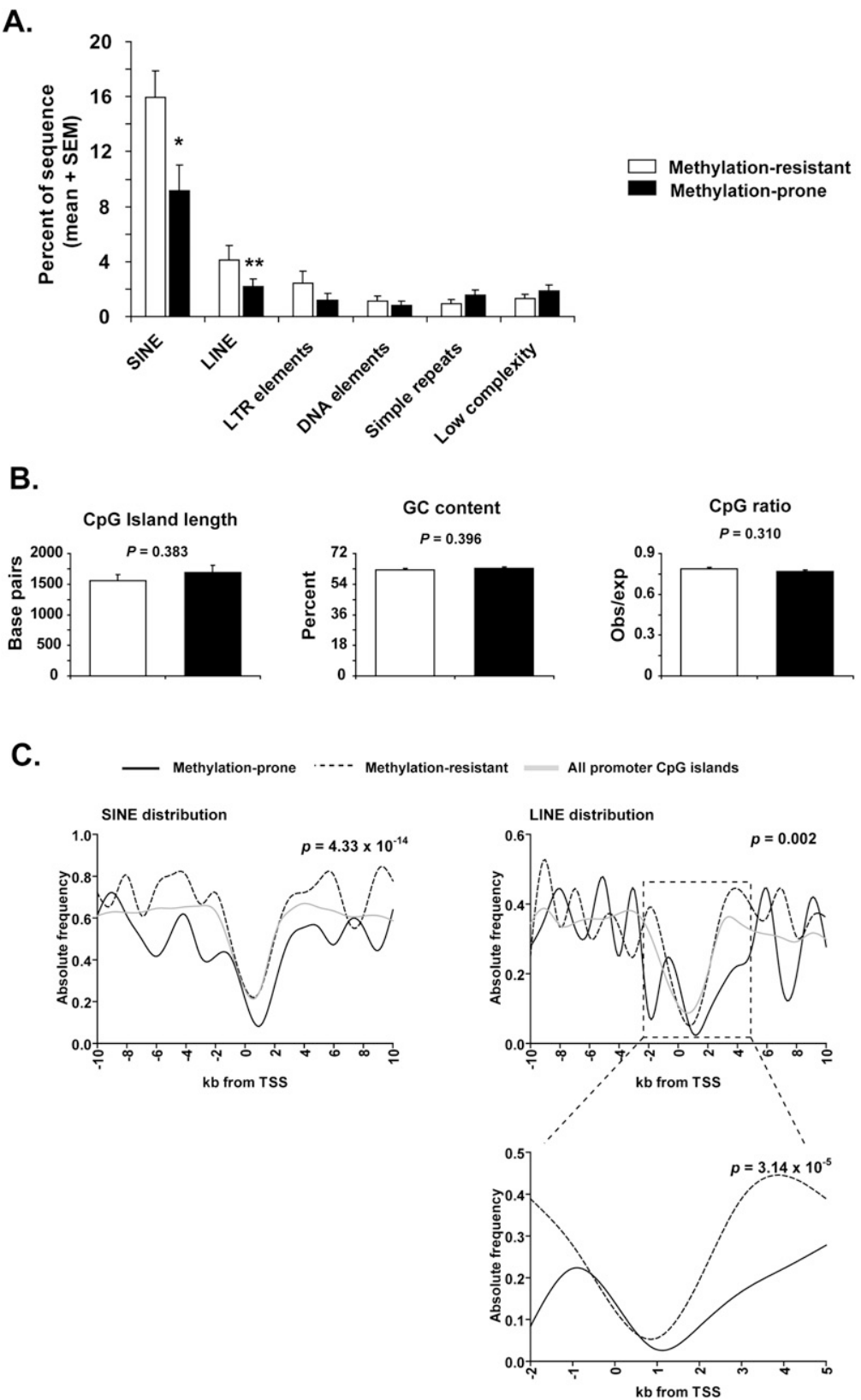

Figure 1. Distribution of repetitive elements in methylation-prone versus methylation-resistant genes. (A) The abundance of repetitive elements of different classes was determined for the 4-kb sequence window centered in the TSS of 36 methylation-resistant (white) and 36 methylation-prone (black) genes. Retrotranposons of the SINE and LINE classes were found to be depleted in methylation-prone genes. ${ }^{*} P<$ 0.02 ; ${ }^{*} P<0.12$ (Student's $t$-test). (B) Average length, GC content, and CpG ratio of CpG islands were not significantly different between methylation-prone and methylation-resistant genes. Error bars represent SEM. (C) Abundance of SINE and LINE retrotransposons in the 20-kb sequence window centered in the TSS of 36 methylation-prone and 36 methylation-resistant genes. The abundance of SINE and LINE retrotransposons in all promoter $\mathrm{CpG}$ islands in the human genome is shown in gray. Note that the depletion of LINE retrotransposons is more significant in the $-2-\mathrm{kb}$ to $+5-\mathrm{kb}$ sequence window.

allowed us to quantify the similarity in distribution of SINE and LINE retrotransposons in a single gene promoter compared to the average distribution of these elements in methylation-prone and methylation-resistant genes. As a result, we could distinguish three groups in the training set: (1) genes depleted of both SINE and LINE repeats, thus predicted to be methylation-prone; (2) genes enriched for SINE and LINE repeats, predicted to be methylation-resistant; and

\section{Genome Research www.genome.org}


A.

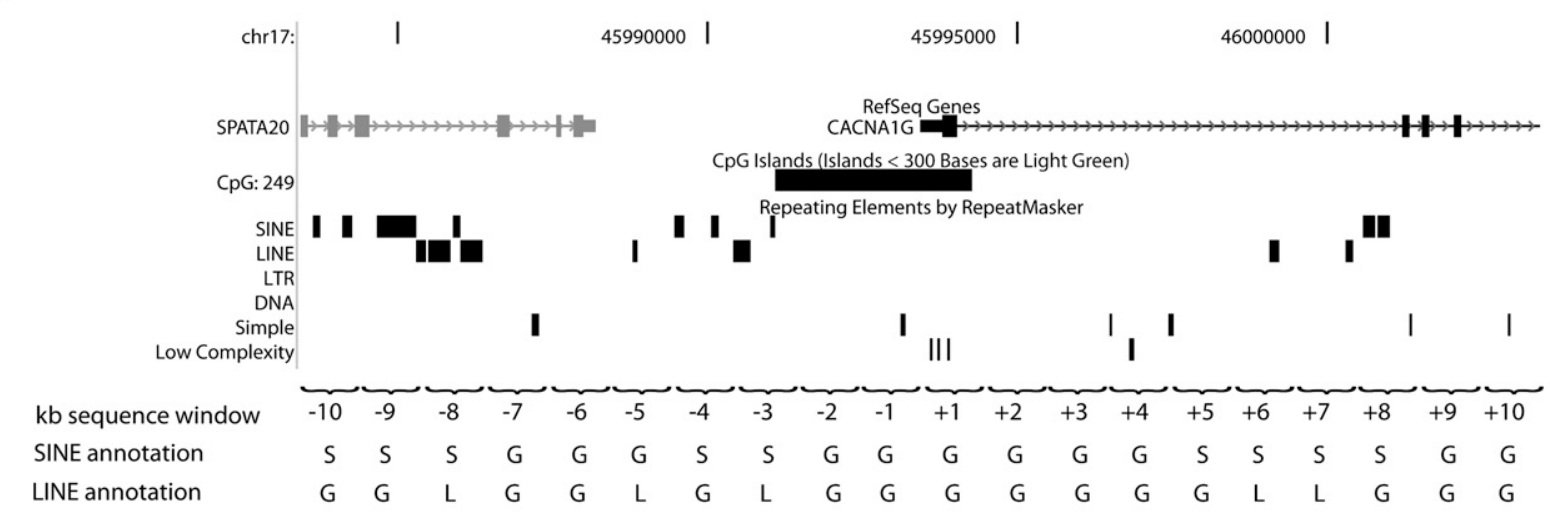

B.

C.

Repetitive element count

Examples of methylation-prone genes

CABIN1 S S G S G G S G G G G G G S S G G G G FOXN3 1 S S S S S S S G G G G G S S S G S G S S MME MDH $S$ S S S S S S S CDH1 SOS $S$ S $S$ S $S$ S

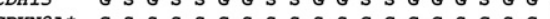
CDKN2A* G S VCAN G S G G G G G G S G G G G G G S G G G DAPK1 S S S G G G S S S S G G G S S G S S

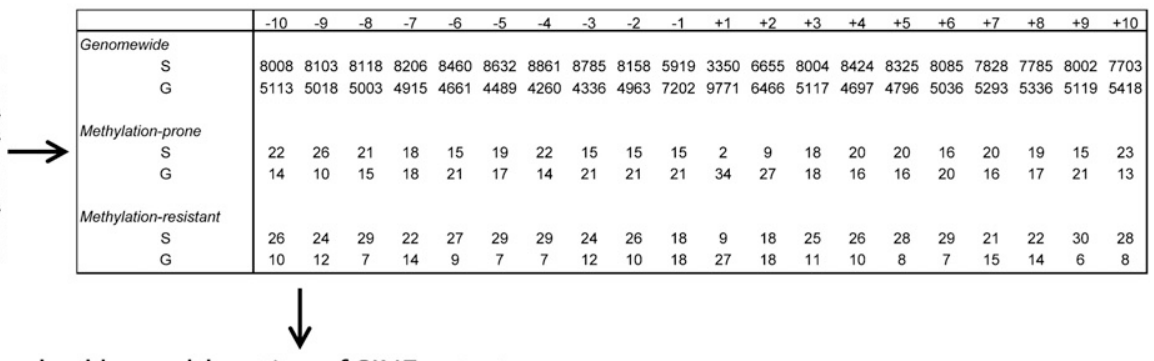

Standard log-odds ratios of SINE retrotransposons

\begin{tabular}{|c|c|c|c|c|c|c|c|c|c|c|c|c|c|c|c|c|c|c|c|c|}
\hline & -10 & -9 & -8 & -7 & -6 & -5 & -4 & -3 & -2 & -1 & +1 & +2 & +3 & +4 & +5 & +6 & +7 & +8 & +9 & +10 \\
\hline \multicolumn{21}{|l|}{ Methylation-prone } \\
\hline$s$ & 0.0287 & 0.1621 & -0.0415 & -0.1936 & -0.3960 & -0.1693 & -0.0381 & -0.4143 & -0.3770 & -0.0631 & -1.3917 & -0.6731 & -0.2025 & -0.1540 & -0.1259 & -0.3161 & -0.0600 & -0.1126 & -0.3433 & 0.1092 \\
\hline G & 0.0254 & -0.2530 & 0.1331 & 0.3161 & 0.5156 & 0.3098 & 0.1405 & 0.5508 & 0.4810 & 0.0978 & 0.2543 & 0.4604 & 0.3315 & 0.3174 & 0.2621 & 0.4351 & 0.1494 & 0.2131 & 0.4242 & -0.1020 \\
\hline \multicolumn{21}{|l|}{ Methylation-resistant } \\
\hline $\mathrm{s}$ & 0.1916 & 0.0840 & 0.2733 & 0.0009 & 0.1732 & 0.2422 & 0.2312 & 0.0398 & 0.1558 & 0.1143 & 0.0215 & -0.0075 & 0.1165 & 0.1009 & 0.2016 & 0.2613 & -0.0126 & 0.0297 & 0.3301 & 0.3015 \\
\hline G & -0.3003 & -0.0766 & -0.6016 & 0.0706 & -0.3098 & -0.5488 & -0.5288 & 0.0031 & -0.2423 & -0.0522 & 0.0294 & 0.0637 & -0.1484 & -0.1406 & -0.4099 & -0.5819 & 0.0865 & 0.0237 & -0.7841 & -0.5685 \\
\hline
\end{tabular}

Substitution matrix of SINE retrotransposons

\begin{tabular}{|c|c|c|c|c|c|c|c|c|c|c|c|c|c|c|c|c|c|c|c|c|}
\hline & -10 & -9 & -8 & -7 & -6 & -5 & -4 & -3 & -2 & -1 & +1 & +2 & +3 & +4 & +5 & +6 & +7 & +8 & +9 & +10 \\
\hline S & -0.1629 & 0.0781 & -0.3148 & -0.1945 & -0.5693 & -0.4114 & -0.2693 & -0.4541 & -0.5328 & -0.1774 & -1.4132 & -0.6655 & -0.3189 & -0.2549 & -0.3274 & -0.5775 & -0.0474 & -0.1424 & -0.6734 & 1923 \\
\hline G & 0.3257 & -0.1763 & 0.7347 & 0.2455 & 0.8254 & 0.8586 & 0.6693 & 0.5477 & 0.7232 & 0.1500 & 0.2250 & 0.3967 & 0.4799 & 0.4579 & 0.6720 & 1.0170 & 0.0629 & 0.1894 & 1.2083 & 4665 \\
\hline
\end{tabular}

Figure 2. SINE and LINE abundance score to predict gene predisposition to methylation in cancer. ( $A$ ) Annotation of SINE and LINE retrotransposons near the promoter sequence of a representative methylation-prone gene (in this example, the CACNA1G gene). The promoter sequence was divided into 20 bins of 1-kb sequence each (10 bins upstream and 10 bins downstream of each gene TSS), and the presence of SINE and LINE retrotransposons was annotated for each bin. Note that each element was annotated to just one bin (the closest to TSS). The same procedure was followed for all human genes with CpG islands overlapping or no more than $200 \mathrm{bp}$ from their TSS. (B) Example of a 20-letter acronym representing SINE retrotransposon abundance in a collection of methylation-prone genes. (C) Counting of SINE presence (S) and absence $(G)$ in all human genes with a promoter CpG island (genome-wide) and the training set of methylation-prone and methylation-resistant genes. SINE abundance was converted to standard log-odds ratios, as described in the Methods section, and the final substitution matrix for SINE retrotransposons is presented (bottom table). The same calculation was done for LINE retrotransposons. *Transcript variant coding for the P16INK4A protein.

(3) genes that were depleted of only one type of repeat. Comparison of these findings with the methylation data for each one of the 72 studied genes revealed that 19 of 23 genes (83\%) predicted to be methylation-prone were actually hypermethylated in cancer and 23 of 25 genes (92\%) predicted to be methylation-resistant were not or were rarely hypermethylated in cancer (Fig. 3A). Genes with discordant frequency of SINE and LINE repeats (30 of 72, 42\%) seemed to represent a class of genes of intermediate predisposition to methylation. We observed that the distribution of retrotransposons in the core promoter $(-1 \mathrm{~kb}$ to $+2 \mathrm{~kb}$ centered in the TSS) is also predictive of methylation predisposition, however with less accuracy than the $20-\mathrm{kb}$ region classification $(75 \%$ of predicted methylation- prone genes were indeed methylated in cancer, and $63 \%$ of the methylation-resistant genes were unmethylated).

\section{Validation of the model}

A major drawback of our model is the limited data set used in its development. We therefore focused on validation using progressively larger data sets. We first studied 74 methylation-prone and 68 methylation-resistant genes for which data on promoter methylation in cancer were available (Supplemental Tables S3, S4) from a variety of tissues (colon, lung, breast, and leukemia, among others). As shown in Figure 3B, $81 \%$ of the predicted methylation-prone 
A.

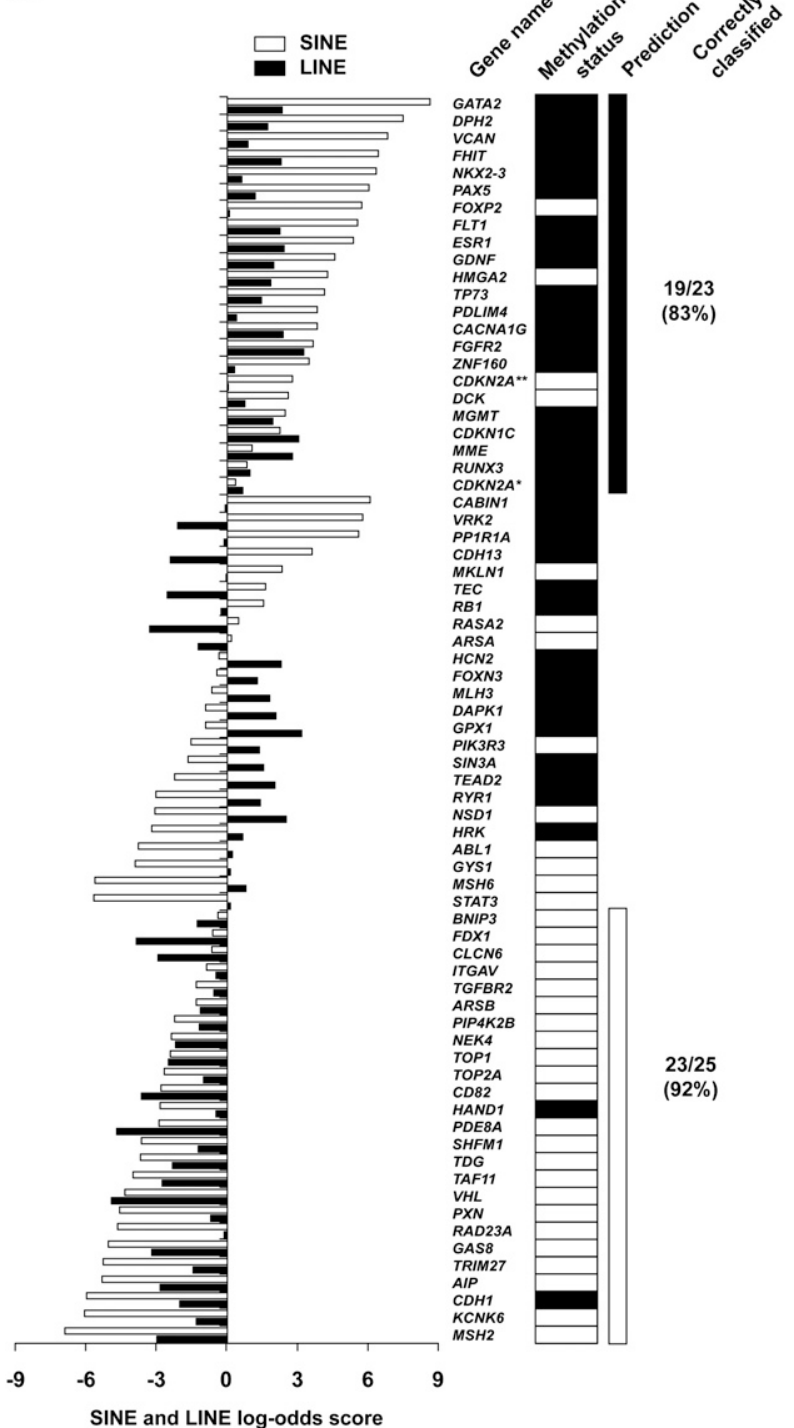

B. Known methylation status
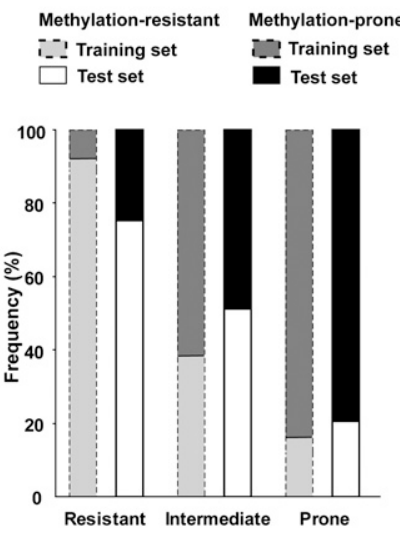

Prediction group

C.

Prediction group

$\square$ Methylation-resistant

Methylation-intermediate

Methylation-prone

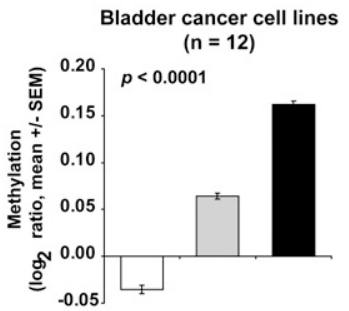

Bladder cancer patients ( $n=28$ )

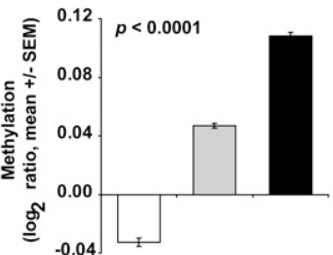

Figure 3. Prediction of gene predisposition and resistance to hypermethylation in cancer. (A) SINE and LINE scores of the training set genes. The scores were calculated according to the described log-odds ratio method for each gene and are represented as horizontal bars (white bars, SINE score; black bars, LINE score). Methylation status determined by bisulfite PCR methods is shown on the right. Genes with concordant depletion of SINE and LINE retrotransposons (log-odds $\geq 0$ ) were predominantly methylation-prone, with the opposite found for genes with enrichment of both SINE and LINE repeats. Discordant SINE and LINE scores likely represent a class of genes with intermediate predisposition to methylation. ${ }^{*}$ Transcript variant coding for the P16INK4A protein. ${ }^{* *}$ Transcript variant coding for the P14ARF protein. Black rectangles represent methylated genes; white rectangles represent unmethylated genes. (B) The predictive method based on SINE and LINE retrotransposons abundance was applied to a test set composed of 142 genes. The frequency of genes correctly classified according to their DNA methylation status in cancer was $79 \%$ for methylation-resistant and $75 \%$ for methylation-prone genes. These values were closely related to those found in the training set (gray bars). (C) Validation of the predictive method in a large set of cancer cell lines and primary cancer tissues. Methylation status of more than 6600 autosomal gene promoters was determined by MCAM. X chromosome genes were excluded from this analysis due to their hemimethylated status in female samples. The measured DNA methylation per tissue type was significantly higher in predicted methylation-prone genes than in predicted methylation-resistant and methylation-intermediate genes. Methylation is presented as the $\log _{2}$ ratio (cancer/control) of all oligonucleotide probes of a predicted methylation status.

genes were indeed methylated in cancer, and $75 \%$ of the predicted methylation-resistant genes were not methylated. As expected, we found a 1:1 ratio of methylated to unmethylated genes in the predicted methylation-intermediate group (52 of 142 genes, 37\%). We further evaluated whether our predictive model held true in a larger scale analysis. For this, we used methylation data from 28 cancer cell lines and 32 primary tissues analyzed by methylated CpG island amplification microarray (MCAM), a sensitive and specific microarray method based on selective amplification of methylated DNA after restriction enzyme digestion (Estecio et al. 2007; Shen et al. 2007b). As performed, MCAM detects cancer-specific methylation as a result of the cohybridization of methylation libraries of cancer versus normal tissues. Thus, all promoter $\mathrm{CpG}$ islands identified by MCAM as methylated in cancer are not methylated in the normal control. In this large data set, composed of more than 26,000 probes representing around $6600 \mathrm{CpG}$ islands associated with autosomal gene promoters, we found that predicted methylation-prone genes showed the highest average values of measured promoter

\section{Genome Research} www.genome.org 
methylation, and the predicted methylation-resistant genes had the lowest values (Fig. 3C). This pattern was consistent across individual samples and tissue types, and was observed in 27 of 28 cancer cell lines (98\%) and in all 32 studied primary cancer tissue samples (Supplemental Fig. S2). Among 740 genes concordantly methylated across tumor samples (i.e., methylated in at least $30 \%$ of the primary tumors and cell lines), 16\% were predicted methylation-resistant, $41 \%$ methylation-intermediate, and $43 \%$ methylation-prone. Thus, our predictive method can accurately classify genes in methylation predisposition groups. In addition, despite the fact that it was generated from cancer cell lines, the predictive model correctly predicts methylation predisposition in primary, uncultured cancer cells.

Although structurally different between mice and humans, retrotransposons are associated with repressed chromatin organization in both species. Thus, if our model is correct, promoter CpG islands subjected to hypermethylation in animal models should also be depleted of retrotransposons. To address this question, we compared the distribution of SINE and LINE retrotransposons in a 20-kb region around the TSS of more than 6000 mouse promoter CpG islands identified by MCAM analysis as methylation-prone and methylation-resistant in a mouse model for myelodysplastic syndrome (Lin et al. 2005). Similar to human cancer, SINE repeats were consistently depleted in methylation-prone genes (Fig. 4A). However, the difference in frequency of LINE repeats was not statistically significant between methylation-prone and methylationresistant genes. Interestingly, the analysis of distribution of LTR repeats revealed that these transposons were less frequent in methylation-prone genes. IAP repeats, a family of LTR repeats highly successful in mice but not present in humans, also showed a trend for depletion in methylation-prone genes. The lack of statistical significance for the differential distribution of IAP repeats between the methylation-predisposition groups is likely due to their low frequency close to gene TSS $(<1 \%$ of gene promoter CpG islands). The same general pattern of frequency of SINE, LINE, and LTR repeats was also observed in an analysis of two additional recently published methylation data sets (Fig. 4B,C) generated from chronic lymphocytic leukemia (CLL) and intestinal cancer mouse models (Hahn et al. 2008; Chen et al. 2009). Additionally, since age-related methylation accounts for a large fraction of promoter CpG island methylation observed in cancer (Toyota and Issa 1999), we reasoned that age-related methylated genes are also likely to be depleted of retrotransposons. The DNA methylation pattern of mouse small intestine tissue was compared between old (35-mo-old) and young (3-mo-old) animals using MCAM. We found that, similar to human and mouse promoter CpG islands predisposed to methylation in cancer, age-related methylated mouse promoter CpG islands were depleted of SINE, LINE, and LTR repeats (Fig. 4D). Thus, our data clearly demonstrate that genes predisposed to age-related methylation share a common genome architecture with cancer-related methylated genes. Based on these findings, we revisited the distribution of LTR elements in our large MCAM data set for human cancers and found that LTRs are also depleted in methylation-prone genes, albeit to a lesser degree than SINE and LINE retrotransposons (Supplemental Fig. S3). However, adding LTR distribution to predict gene predisposition to DNA methylation in human cancer did not significantly improve our model.

\section{Genome-wide analysis of methylation predisposition}

The validation of our predictive model prompted us to apply it genome-wide. Among 25,489 unique RefSeq genes (NCBI Build $36.1), 16,166$ (63.4\%) have a promoter CpG island. Of these, 3613
(22.3\%) were predicted by our model to be methylation-prone; $7308(45.2 \%)$ were predicted to be moderately predisposed to methylation (methylation-intermediate); and the remaining 5245 (32.5\%) were predicted to be methylation-resistant (Fig. 5A).

The top 50 predicted methylation-prone and methylationresistant genes are presented in Tables 1 and 2. Forty-eight percent ( 24 of 50) of the top predicted methylation-prone genes have been described as methylated in cancer according to data in the literature, and only $6 \%$ (3/50) of the top predicted methylation-resistant genes were described as methylated. Although not present in Table 1 because they did not rank among the top 50 genes, several classical genes known to be methylated in cancer and not previously included in the first validation set were correctly predicted as methylation-prone (for example RASSF1, GATA4, GATA5, and SFRP2). In terms of gene function, 39/50 (78\%) predicted methylation-prone genes are directly related to developmental processes, compared to only $5 \%$ of the predicted methylationresistant genes $(P<0.001$, Fisher's exact test). Indeed, when applied genome-wide, Gene Ontology analysis revealed that methylationprone genes preferentially participate in developmental processes (Fig. 5B), an observation in agreement with previous data showing that repeat-free regions in mammalian genomes are enriched for genes that function in organogenesis and morphogenesis, among other functions related to development (Simons et al. 2006). Some of these are multiclusters of neighboring genes with related function, such as $H O X$ gene clusters. This prompted us to quantify the propensity of predicted methylation-prone genes to be correlated in neighboring genes and to find 28 genomic regions with statistically significant higher frequency of such genes (Supplemental Table S5). These regions were in average $5 \mathrm{Mb}$ long (ranging from 0.3 to $26 \mathrm{Mb}$ ) and had a tendency to be located close to telomeric regions. Together, these regions cover $\sim 4.5 \%$ of the human genome but contain $15 \%$ of the predicted methylationprone genes. Twelve (43\%) of these regions included multiclusters of genes with similar function and likely originated from duplication events.

Two classes of genes with methylated promoter CpG islands in normal tissues are imprinted and X chromosome-inactivated genes. Among 30 well-characterized imprinted genes, 12 (40\%) were predicted methylation-prone, 17 (57\%) were predicted intermediate, and only one was predicted methylation-resistant (Supplemental Table S6). The frequency of predicted methylationprone, resistant, and intermediate genes on the X chromosome was remarkably similar to the frequency of the predicted classes genome-wide. There was a moderate, although not significant, enrichment of predicted methylation-prone genes among genes that escape $\mathrm{X}$ inactivation ( $P>0.05, \chi^{2}$ test, Supplemental Fig. S4).

Although we have focused our analysis on promoter CpG islands, nonpromoter $\mathrm{CpG}$ islands (both exonic/intronic and intergenic $\mathrm{CpG}$ islands) are also subject to de novo methylation in cancer. There are scarce data on these $\mathrm{CpG}$ islands in the literature, but we were able to identify 291 nonpromoter CpG islands in our MCAM database that showed frequent hypermethylation in cell lines and primary tumors, and 740 methylation-resistant nonpromoter CpG islands. Similarly to promoter CpG islands, methylation-prone nonpromoter $\mathrm{CpG}$ islands were found to be depleted of SINE and LINE retrotransposons (Supplemental Fig. S5). Although the functional role of these $\mathrm{CpG}$ islands remains poorly defined, our data suggest that the underlying genomic architecture is closely related between methylation-prone and methylationresistant $\mathrm{CpG}$ islands regardless of their position relative to transcriptional units. 
A.

MDS mouse model
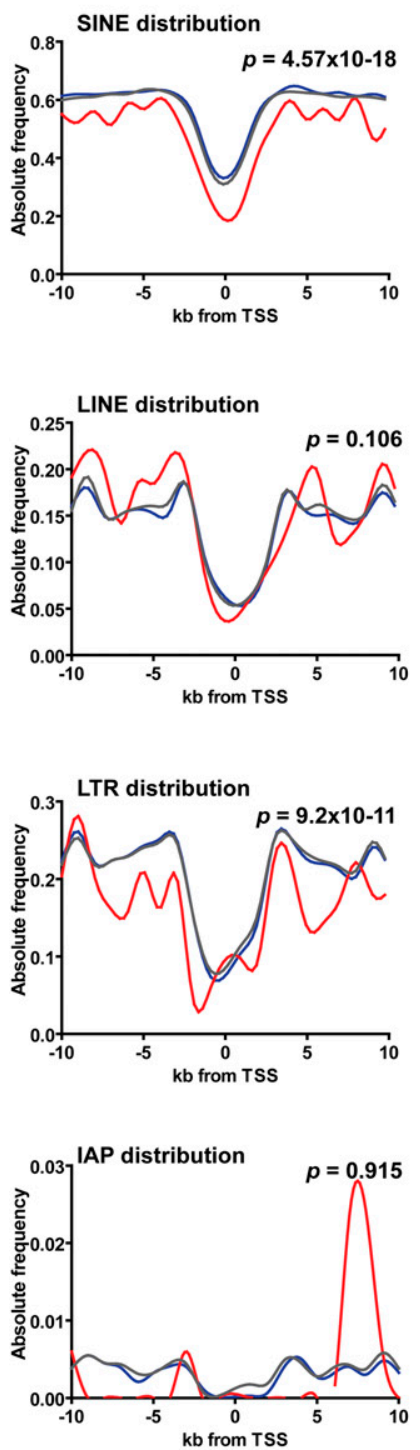

B.

\section{CLL mouse model}
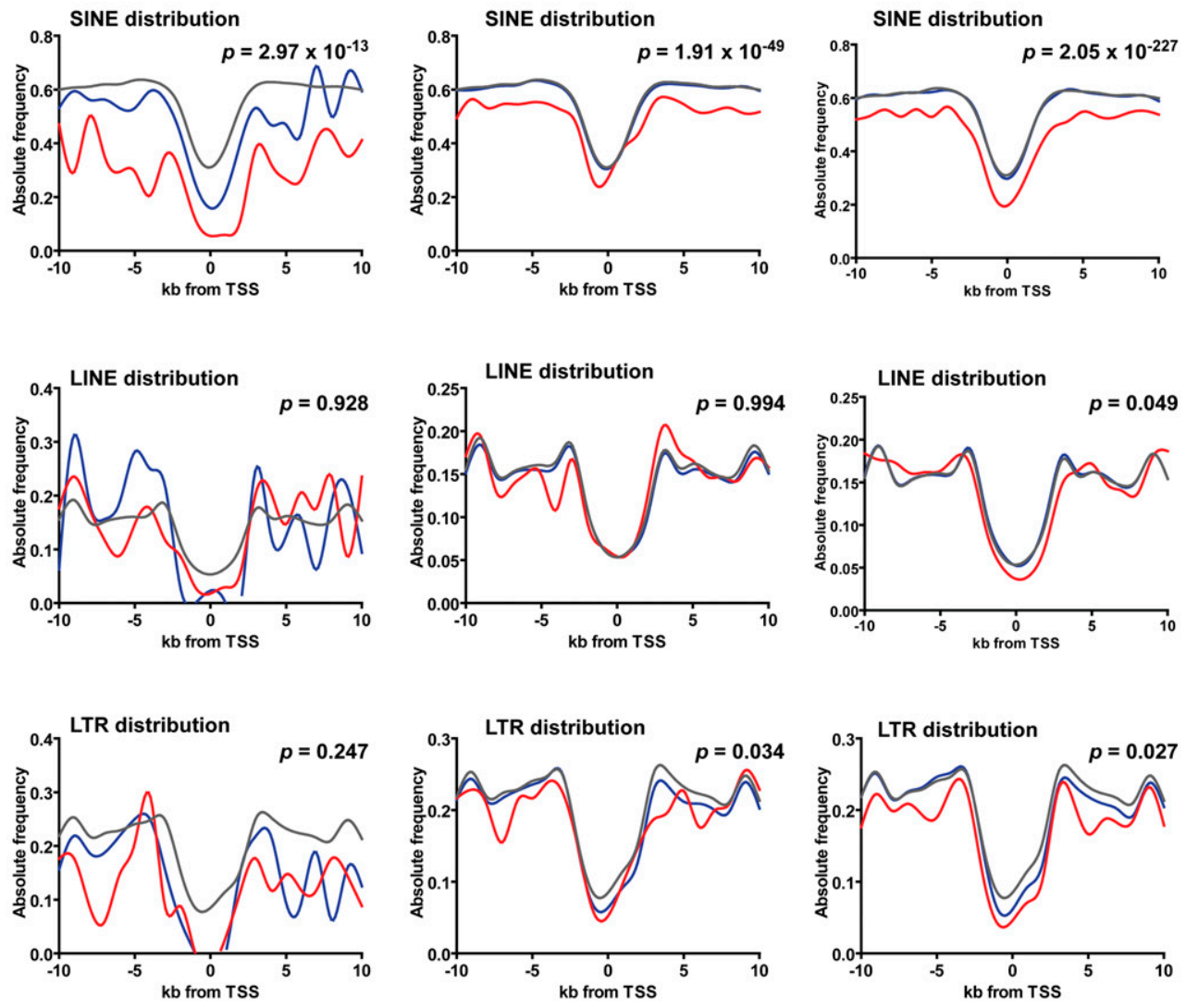

D.

\section{Young versus old mice}

SINE distribution
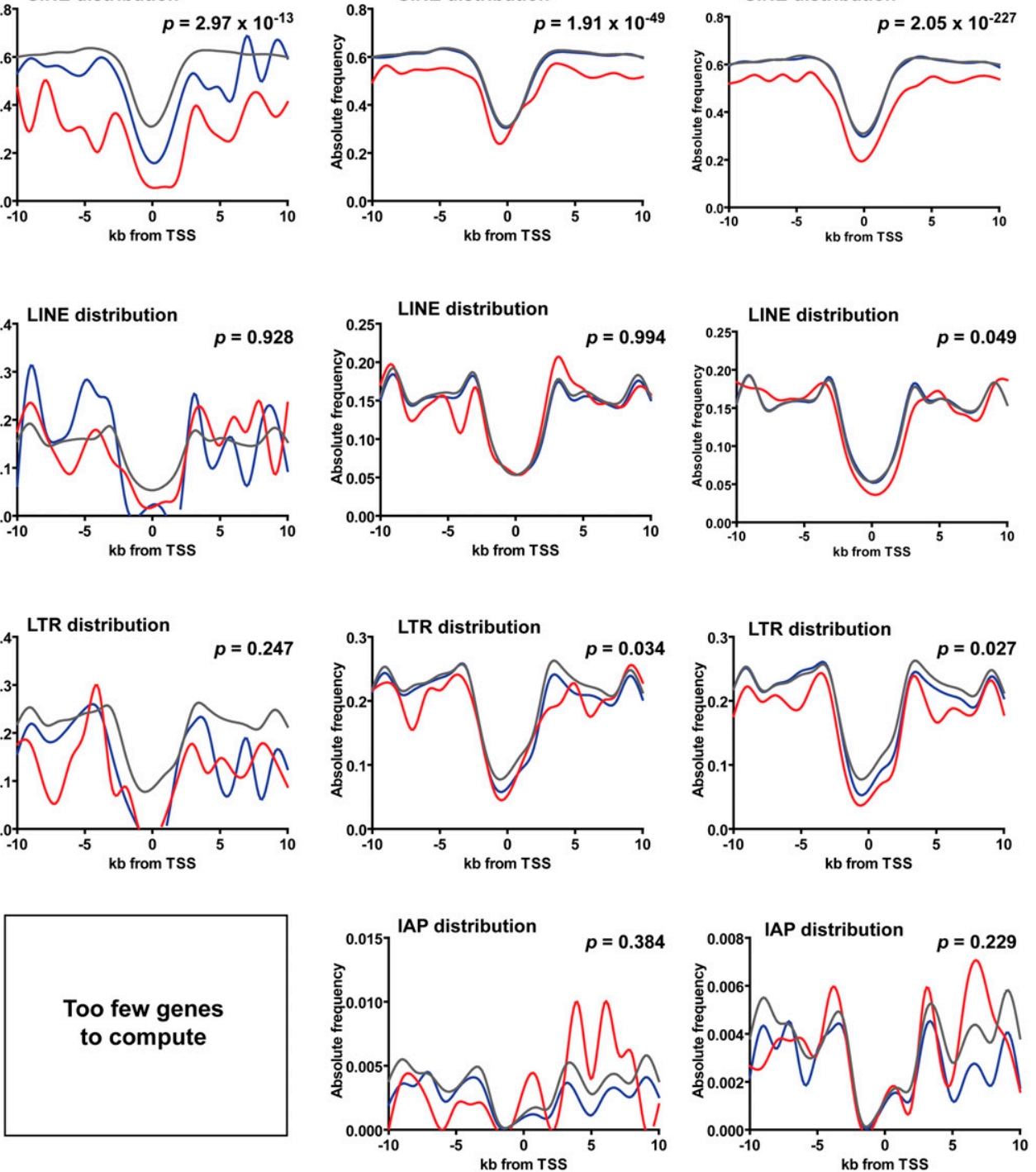

- Methylation-prone

\section{Methylation-resistant}

\section{All promoter CGIs}

Figure 4. Frequency of retroelements in methylation-prone and methylation-resistant genes identified in mouse cancer models and old mice. ( $A$ ) Depletion of SINE and LTR but not LINE repeats near TSS marks methylated promoter CpG islands in a mouse model of myelodysplastic syndrome (MDS). Bone marrow samples of three NUP98-HOXD13 transgene animals that developed MDS (Lin et al. 2005) were studied by MCAM. Bone marrow samples from nontransgene animal of the same mouse strain was used as control, and the methylation status of approximately $6000 \mathrm{CpG}$ island promoter genes was determined in the MCAM experiments. $(B, C)$ The same pattern of retroelements depletion is observed in hypermethylated genes in CLL (Chen et al. 2009) and intestinal cancer mouse models (Hahn et al. 2008). (D) Depletion of SINE, LINE, and LTR repeats near TSS also marks age-related methylation promoter CpG islands. Small intestine tissue harvested from young (3-mo-old) and old (35-mo-old) C57BL/6J mice were used in MCAM experiments to identify age-related methylation.

\section{Effects on gene expression}

To validate the biological implications of our predictive model, we compared the mRNA expression of predicted methylation-prone and methylation-resistant promoter CpG island genes in 28 normal differentiated human tissues and 52 human cancer cell line samples using public microarray databases (Ross et al. 2000; Su et al. 2004). As shown in Figure 5C, in normal tissues, predicted methylation-resistant genes had in general lower expression than predicted methylation-prone genes. An attractive explanation for the lower expression of predicted methylation-resistant genes in normal cells is their higher content of retrotransposons, which have been implicated in decreased mRNA expression through impairment of elongation (Han et al. 2004). As would be expected

\section{Genome Research www.genome.org}


A.

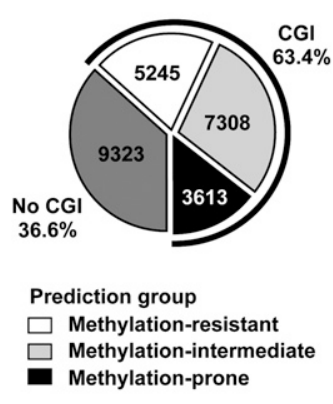

B.

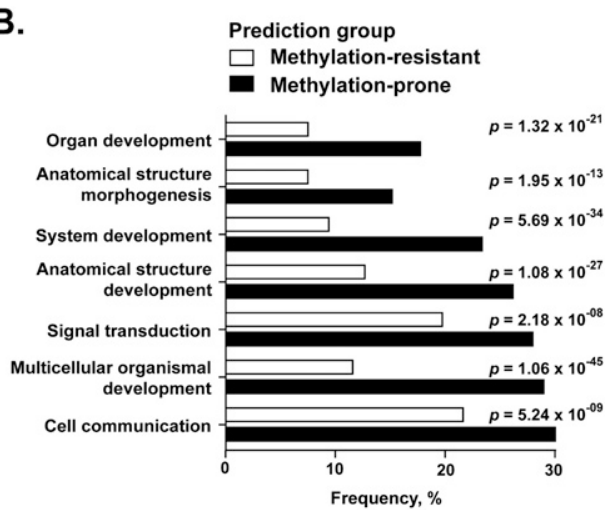

C.

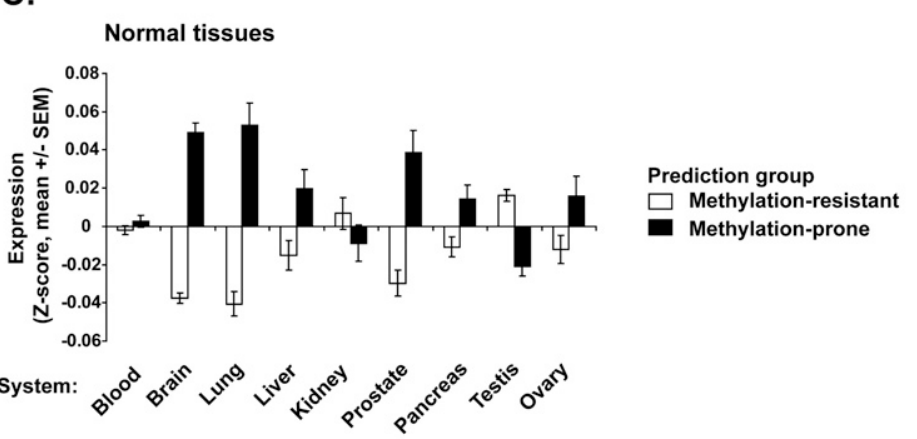

D.

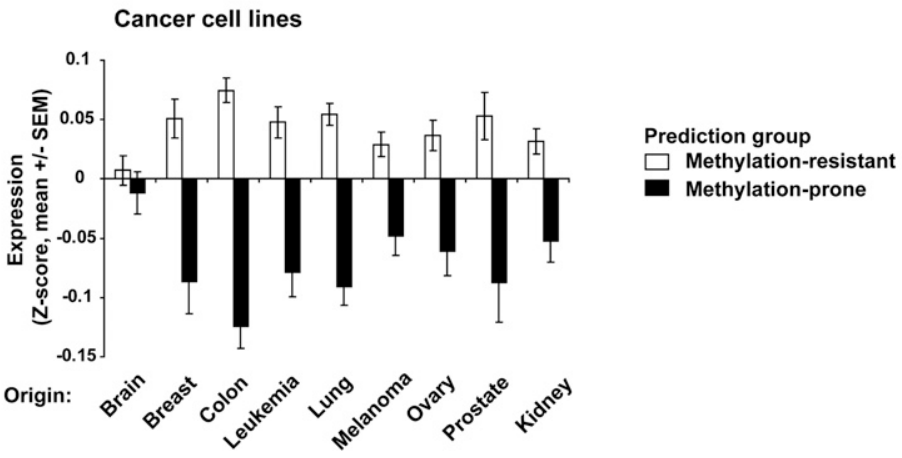

Figure 5. Genome-wide prediction of predisposition to DNA methylation in cancer. $(A)$ The pie chart shows the number of RefSeq genes with no $\mathrm{CpG}$ islands (dark gray) and the number of predicted methylation-resistant (white), methylation-intermediate (light gray), and methylation-prone (black) genes in promoter CpG island genes. (B) Gene Ontology (GO) analysis of 1952 predicted methylation-prone and 2583 predicted methylation-resistant genes for which functional information was available. Horizontal bars represent the frequency of significant GO terms. (C) Gene expression analysis for 2822 promoter $\mathrm{CpG}$ island associated genes predicted methylation-prone and 3651 predicted methylation-resistant genes in normal tissues. Expression values were retrieved from the GNF database (Su et al. 2004) and Z-score normalized per tissue. Each bar represents the mean \pm SEM expression values in each tissue according to their predicted methylation predisposition. $(D)$ Gene expression analysis for 599 promoter $\mathrm{CpG}$ island associated genes predicted methylationprone and 996 predicted methylation-resistant genes in 52 cancer cell lines. Expression values were retrieved from a published work (Ross et al. 2000) and were analyzed as described in C. Only genes present in the studied array platforms could be evaluated, resulting in a different number of analyzed genes in each experiment.

if our predictive model were correct (since hypermethylation of gene promoters results in gene silencing), we observed in cancer cell lines that predicted methylation-prone genes were down-regulated compared to predicted methylation-resistant genes (Fig. 5D). Thus, depletion of retrotransposons near the TSS was found to be an independent predictor of gene down-regulation in cancer.
Comparison with hyperconserved domains and PcG protein marking

Enrichment for developmental genes was previously described (Tanay et al. 2007) among genes associated with hyperconserved $\mathrm{CpG}$ domains, which were identified based on DNA sequence features. Analysis of our data revealed that $84 \%$ of the genes in hyperconserved domains are predicted methylation-prone (Fig. 6A). However, hyperconservation is a feature of a much smaller subset of hypermethylated genes in cancer than depletion of retrotransposons (Fig. 6B). Another feature of hyperconserved domains is the overlap with multicluster gene families, such as $H O X$ genes. Among the identified genomic clusters predicted methylation-prone, 11 (39\%) included hyperconserved domains (Supplemental Table S5).

Hyperconserved domains and DNA methylation have been shown to correlate with Polycomb group (PcG) protein binding in embryonic stem cells. We therefore studied the relationship between our methylation prediction and PcG protein binding. First, we compared the presence of H3K27me3 in predicted methylationprone and methylation-resistant genes according to chromatin immunoprecipitation microarray results for 8727 gene promoters in three cell lines: PC3, a prostate cancer cell line; MCF7, a breast cancer cell line; and PrEC, an immortalized normal prostate epithelial cell line (Kondo et al. 2008). As shown in Figure 6C, predicted methylation-prone genes were enriched for H3K27me3 in all three cell lines tested. We next evaluated the frequency of binding of PcG proteins and H3K27me3 to genes predicted to be methylationprone or methylation-resistant according to the occupancy maps published for embryonic stem cells (Lee et al. 2006). Genes predicted to be methylation-prone had a higher frequency of SUZ12, EED, and H3K27me3 targets compared to genes predicted to be methylation-resistant (Fig. 6D; Supplemental Fig. S6A). Indeed, a direct comparison of the distribution of SINE and LINE retrotransposons in genes targeted by Suz12 revealed a large difference, in the same direction as observed between cancer methylation-prone and methylationresistant genes (Supplemental Fig. S6B).

Since PcG protein marking in embryonic stem cells is closely related to predisposition to DNA methylation in cancer (Ohm et al. 2007; Schlesinger et al. 2007; Widschwendter et al. 2007), it is possible that the relationship between retrotransposon abundance and CpG island DNA methylation is a consequence rather than an independent phenomenon from PcG protein marking. In this 
Estécio et al.

Table 1. Top 50 genes predicted as methylation-prone

\begin{tabular}{|c|c|c|c|c|c|c|}
\hline Gene symbol & Gene name & $\begin{array}{l}\text { RefSeq } \\
\text { name }\end{array}$ & Chromosome & $\begin{array}{l}\text { Transcription } \\
\text { start }\end{array}$ & $\begin{array}{c}S \\
\text { score }\end{array}$ & $\begin{array}{c}L \\
\text { score }\end{array}$ \\
\hline ZFPM2 & Zinc finger protein, multitype 2 & NM_012082 & $\operatorname{chr} 8$ & 106400322 & 10.0794 & 2.5599 \\
\hline TOX3 & TOX high mobility group box family member 3 & NM_001080430 & chr 16 & 51138307 & 9.7349 & 2.8928 \\
\hline TPPP & Tubulin polymerization promoting protein & NM_e07030 & $\operatorname{chr} 5$ & 746510 & 10.0794 & 2.4442 \\
\hline FOXA1 & Forkhead box A1 & NM_004496 & chr 14 & 37134240 & 10.3338 & 2.1021 \\
\hline$N K \times 2-2$ & NK2 homeobox 2 & NM_002509 & chr 20 & 21442664 & 9.4206 & 2.9432 \\
\hline TCERG1L & Transcription elongation regulator 1 -like & NM__174937 & chr 10 & 132999974 & 9.0299 & 3.2956 \\
\hline POU3F3 & POU class 3 homeobox 3 & NM_006236 & $\operatorname{chr} 2$ & 104838400 & 10.0794 & 2.2061 \\
\hline DUX4 & Double homeobox, 4 & NM_033178 & $\operatorname{chr} 4$ & 191229360 & 10.0794 & 1.978 \\
\hline LHX9 & LIM homeobox 9 & NM_001014434 & chr 1 & 196148257 & 10.0794 & 1.9779 \\
\hline EBF3 & Early B-cell factor 3 & NM_001005463 & chr 10 & 131652081 & 10.0794 & 1.9779 \\
\hline GATA3 & GATA binding protein 3 & NM_M02051 & chr 10 & 8136672 & 10.0794 & 1.9779 \\
\hline GPR123 & G protein-coupled receptor 123 & NM_001083909 & chr 10 & 134751398 & 10.0794 & 1.9779 \\
\hline IGF2 & Insulin-like growth factor 2 (somatomedin A) & NM_000612 & chr 11 & 2116780 & 10.0794 & 1.9779 \\
\hline PAX6 & Paired box 6 & NM_001604 & chr 11 & 31789434 & 10.0794 & 1.9779 \\
\hline HOXC4 & Homeobox C4 & NM_014620 & chr 12 & 52696908 & 10.0794 & 1.9779 \\
\hline HOXC8 & Homeobox C8 & NM_022658 & chr 12 & 52689156 & 10.0794 & 1.9779 \\
\hline HOXC9 & Homeobox C9 & NM_006897 & chr 12 & 52680143 & 10.0794 & 1.9779 \\
\hline ZIC2 & $\begin{array}{l}\text { Zic family member } 2 \text { (odd-paired homolog, } \\
\text { Drosophila) }\end{array}$ & NM_007129 & chr 13 & 99432319 & 10.0794 & 1.9779 \\
\hline ZIC5 & $\begin{array}{l}\text { Zic family member } 5 \text { (odd-paired homolog, } \\
\text { Drosophila) }\end{array}$ & NM_033132 & chr 13 & 99422179 & 10.0794 & 1.9779 \\
\hline CRIP2 & Cysteine-rich protein 2 & NM_001312 & chr 14 & 105012175 & 10.0794 & 1.9779 \\
\hline SIX1 & SIX homeobox 1 & NM_005982 & chr 14 & 60185933 & 10.0794 & 1.9779 \\
\hline$N R 2 F 2$ & $\begin{array}{l}\text { Nuclear receptor subfamily } 2 \text {, group } F \text {, } \\
\text { member } 2\end{array}$ & NM_021005 & chr 15 & 94674949 & 10.0794 & 1.9779 \\
\hline HOXB4 & Homeobox B4 & NM_024015 & chr 17 & 44010742 & 10.0794 & 1.9779 \\
\hline HOXB5 & Homeobox B5 & NM_002147 & chr 17 & 44026102 & 10.0794 & 1.9779 \\
\hline $\mathrm{ZADH} 2$ & $\begin{array}{l}\text { Zinc binding alcohol dehydrogenase domain } \\
\text { containing } 2\end{array}$ & NM_175907 & chr 18 & 71050105 & 10.0794 & 1.9779 \\
\hline TSHZ3 & Teashirt zinc finger homeobox 3 & NM_020856 & chr 19 & 36532030 & 10.0794 & 1.9779 \\
\hline$D L X 1$ & Distal-less homeobox 1 & NM_178120 & chr 2 & 172658453 & 10.0794 & 1.9779 \\
\hline HOXD10 & Homeobox D10 & NM_002148 & chr 2 & 176689737 & 10.0794 & 1.9779 \\
\hline HOXD11 & Homeobox D11 & NM_021192 & $\operatorname{chr} 2$ & 176680329 & 10.0794 & 1.9779 \\
\hline HOXD12 & Homeobox D12 & NM_021193 & chr 2 & 176672775 & 10.0794 & 1.9779 \\
\hline HOXD8 & Homeobox D8 & NM_019558 & $\operatorname{chr} 2$ & 176702722 & 10.0794 & 1.9779 \\
\hline HOXD9 & Homeobox D9 & NM_014213 & chr 2 & 176695333 & 10.0794 & 1.9779 \\
\hline MEIS1 & Meis homeobox 1 & NM_002398 & $\operatorname{chr} 2$ & 66516035 & 10.0794 & 1.9779 \\
\hline$N R 4 A 2$ & $\begin{array}{l}\text { Nuclear receptor subfamily } 4 \text {, group A, } \\
\text { member } 2\end{array}$ & NM_006186 & $\operatorname{chr} 2$ & 156897446 & 10.0794 & 1.9779 \\
\hline SATB2 & SATB homeobox 2 & NM_015265 & $\operatorname{chr} 2$ & 200033446 & 10.0794 & 1.9779 \\
\hline POU4F2 & POU class 4 homeobox 2 & NM_004575 & $\operatorname{chr} 4$ & 147779494 & 10.0794 & 1.9779 \\
\hline IRX1 & Iroquois homeobox 1 & NM_024337 & $\operatorname{chr} 5$ & 3649167 & 10.0794 & 1.9779 \\
\hline POU3F2 & POU class 3 homeobox 2 & NM_005604 & $\operatorname{chr} 6$ & 99389300 & 10.0794 & 1.9779 \\
\hline$D L X 6$ & Distal-less homeobox 6 & NM_005222 & chr 7 & 96473225 & 10.0794 & 1.9779 \\
\hline HOXA10 & Homeobox A10 (isoform a) & NM_018951 & chr 7 & 27186368 & 10.0794 & 1.9779 \\
\hline HOXA10 & Homeobox A10 (isoform b) & NM_153715 & chr 7 & 27180480 & 10.0794 & 1.9779 \\
\hline HOXA5 & Homeobox A5 & NM_019102 & $\operatorname{chr} 7$ & 27149812 & 10.0794 & 1.9779 \\
\hline HOXA6 & Homeobox A6 & NM_024014 & chr 7 & 27153893 & 10.0794 & 1.9779 \\
\hline HOXA7 & Homeobox A7 & NM_006896 & chr 7 & 27162821 & 10.0794 & 1.9779 \\
\hline HOXA9 & Homeobox A9 & NM_152739 & $\operatorname{chr} 7$ & 27171674 & 10.0794 & 1.9779 \\
\hline$S C R I B$ & Scribbled homolog (Drosophila) & NM_182706 & $\operatorname{chr} 8$ & 144969537 & 10.0794 & 1.9779 \\
\hline$S C X B$ & Scleraxis homolog B (mouse) & NM_001080514 & $\operatorname{chr} 8$ & 145461410 & 10.0794 & 1.9779 \\
\hline NFIB & Nuclear factor I/B & NM̄_005596 & chr 9 & 14303945 & 10.0794 & 1.9779 \\
\hline METRNL & $\begin{array}{l}\text { Meteorin, glial cell differentiation } \\
\text { regulator-like }\end{array}$ & NM_001004431 & chr 17 & 78630855 & 9.1408 & 2.8277 \\
\hline OTP & Orthopedia homeobox & NM_032109 & $\operatorname{chr} 5$ & 76970278 & 9.4805 & 2.4357 \\
\hline
\end{tabular}

case, our predictive model would have no additional predictive value beyond that of PcG protein marking alone. We first examined the relative contribution of PcG marking and our predictive model in genes known to be methylated in our training and first testing set. Out of 110 frequently methylated genes, 25 (23\%) are marked by both PcG and predicted methylation-prone, 31 (28\%) are predicted methylation-prone alone, 15 (14\%) are PcG-positive alone, and $39(35 \%)$ are neither marked by PcG nor predicted methylation-prone (Fig. 6E). Thus, in this set, PcG marking and our predictive model based on retrotransposon frequency appear to be correlated but also complementary. Moreover, these results show that retrotransposon distribution marks a larger fraction of methylation-prone genes than PcG protein marking. To examine this in a larger data set, we turned to MCAM data and divided genes into predicted methylation-prone and methylation-resistant (excluding intermediate for clarity). As seen in Figure $6 \mathrm{~F}$, both predicted methylation-prone and PcG-marked genes have significantly higher levels of measured methylation than methylation-resistant/PcGnegative subsets. Again, PcG marking alone identifies a smaller subset of methylated genes compared to our predictive model alone (119

\section{Genome Research


Table 2. Top 50 genes predicted as methylation-resistant

\begin{tabular}{|c|c|c|c|c|c|c|}
\hline Gene symbol & Gene name & $\begin{array}{l}\text { RefSeq } \\
\text { name }\end{array}$ & Chrom & $\begin{array}{l}\text { Transcription } \\
\text { start }\end{array}$ & $\begin{array}{c}S \\
\text { score }\end{array}$ & $\begin{array}{c}L \\
\text { score }\end{array}$ \\
\hline SMYD4 & SET and MYND domain containing 4 & NM_052928 & chr 17 & 1679925 & -7.6213 & -7.5946 \\
\hline SMN2 & Survival of motor neuron 2 , centromeric & NM_022877 & $\operatorname{chr} 5$ & 70256523 & -7.5439 & -6.2266 \\
\hline SMN1 & Survival of motor neuron 1 , telomeric & NM_000344 & chr 5 & 70256523 & -7.5439 & -6.2266 \\
\hline PPIL2 & Peptidylprolyl isomerase (cyclophilin)-like 2 & NM_148176 & chr 22 & 20350272 & -6.4342 & -6.9256 \\
\hline RBM44 & RNA binding motif protein 44 & NM_001080504 & chr 2 & 238372126 & -5.5751 & -7.7633 \\
\hline NOSIP & Nitric oxide synthase interacting protein & NM̄_015953 & chr 19 & 54775615 & -6.8053 & -6.3264 \\
\hline ZFP1 & Zinc finger protein 1 homolog (mouse) & NM_153688 & chr 16 & 73739921 & -7.6213 & -5.5062 \\
\hline PXMP4 & Peroxisomal membrane protein $4,24 \mathrm{kDa}$ & NM_007238 & chr 20 & 31771797 & -6.8522 & -6.1378 \\
\hline NHP2L1 & $\begin{array}{l}\text { NHP2 non-histone chr omosome protein } 2 \text {-like } 1 \\
\text { (S. cerevisiae) }\end{array}$ & NM_005008 & chr 22 & 40408502 & -7.6213 & -5.3096 \\
\hline$D R G 1$ & Developmentally regulated GTP binding protein 1 & NM_004147 & chr 22 & 30125538 & -7.2939 & -5.3636 \\
\hline RPA1 & Replication protein $\mathrm{A} 1,70 \mathrm{kDa}$ & NM 002945 & chr 17 & 1680094 & -7.2939 & -5.3193 \\
\hline JAGN1 & Jagunal homolog 1 (Drosophila) & NM_032492 & chr 3 & 9907271 & -6.8225 & -5.5777 \\
\hline EP400 & E1A binding protein p400 & NM 015409 & chr 12 & 131000460 & -6.7769 & -5.4792 \\
\hline PAAF1 & Proteasomal ATPase-associated factor 1 & NM_025155 & chr 11 & 73265680 & -7.6213 & -4.5654 \\
\hline TRPV 4 & $\begin{array}{l}\text { Transient receptor potential cation channel, subfamily } \\
\text { V, member } 4\end{array}$ & NM_147204 & chr 12 & 108755595 & -7.6213 & -4.51 \\
\hline CDK5R & CDK5 regulatory subunit associated protein 2 & NM_018249 & chr 9 & 122382258 & -7.6213 & -4.4664 \\
\hline$I Q C D$ & IQ motif containing D & NM_138451 & chr 12 & 112143263 & -6.2945 & -5.7063 \\
\hline C12orf32 & Chromosome 12 open reading frame 32 & NM_031465 & chr 12 & 2856649 & -6.2921 & -5.683 \\
\hline CYBSRL & Cytochr ome b5 reductase-like & NM_001031672 & chr 1 & 54438334 & -6.5718 & -5.3888 \\
\hline NPRL3 & Nitrogen permease regulator-like 3 (S. cerevisiae) & NM_001039476 & chr 16 & 128672 & -7.6213 & -4.2671 \\
\hline CDK5RAP1 & CDK5 regulatory subunit associated protein 1 & NM_016408 & chr 20 & 31452998 & -7.1813 & -4.6612 \\
\hline CCDC101 & Coiled-coil domain containing 101 & NM 138414 & hr 16 & 28472757 & -6.9085 & -4.9031 \\
\hline CHCHD8 & Coiled-coil-helix-coiled-coil-helix domain containing 8 & NM_016565 & chr 11 & 73265538 & -7.2895 & -4.4949 \\
\hline SLC24A6 & $\begin{array}{l}\text { Solute carrier family } 24 \text { (sodium/potassium/calcium } \\
\text { exchanger), member } 6\end{array}$ & NM_024959 & chr 12 & 112257308 & -7.6213 & -4.1074 \\
\hline DHX37 & DEAH (Asp-Glu-Ala-His) box polypeptide 37 & NM_032656 & chr 12 & 124039620 & -7.6213 & -4.0842 \\
\hline$D N A / C 8$ & Dnal (Hsp40) homolog, subfamily C, member 8 & NM_014280 & & 28432129 & -7.6213 & -4.0769 \\
\hline ZNF562 & Zinc finger protein 562 & NM_017656 & chr 19 & 9646734 & -7.8757 & -3.8041 \\
\hline$D R G 2$ & Developmentally regulated GTP binding protein 2 & NM_001388 & hr 17 & 17932007 & -5.7396 & -5.8857 \\
\hline C16orf45 & Chromosome 16 open reading frame 45 & NM_033201 & chr 16 & 15435825 & -5.9831 & -5.6375 \\
\hline PLA2G16 & Phospholipase A2, group XVI & NM 007069 & & 63138469 & -7.8757 & -3.6817 \\
\hline FOXR1 & Ox R1 & 81721 & chr 11 & 118347626 & -5.4078 & -6.0503 \\
\hline KIF3A & member $3 \mathrm{~A}$ & NM_007054 & chr 5 & 132101164 & -6.4861 & -4.9674 \\
\hline RNF18. & r protein 185 & & r 22 & & -7.6213 & -3.8222 \\
\hline MRPL37 & mal protein L37 & NM_016491 & chr 1 & 54438427 & -6.2901 & -5.1153 \\
\hline YIPF1 & Yip1 domain family, member 1 & NM_018982 & chr 1 & 54128041 & -4.4365 & -6.8572 \\
\hline RAD51L3 & RAD51-like 3 (S. cerevisiae) & NM_002878 & hr 17 & 30471001 & -7.1327 & -4.1404 \\
\hline DNAL1 & light chain 1 & NM_031427 & chr 14 & 73181454 & -5.9831 & -5.265 \\
\hline HLCS & $\begin{array}{l}\text { Holocarboxylase synthetase [biotin-(proprionyl- } \\
\text { CoA-carboxylase [ATP-hydrolyzing]) ligase] }\end{array}$ & NM_000411 & chr 21 & 37284373 & -5.6994 & -5.5373 \\
\hline MMP24 & Matrix metallopeptidase 24 (membrane-inserted) & NM_006690 & & & -6.7495 & -4.4684 \\
\hline MRPL1 & Mitochondrial ribosomal protein L1 & & & & -5 & -5.7917 \\
\hline SETDB1 & SET domain, bifurcated 1 & NM 012432 & chr 1 & 149165511 & -6.9085 & -4.291 \\
\hline CTNNA1 & Catenin (cadherin-associated protein), alpha 1, $102 \mathrm{kDa}$ & NM_001903 & chr 5 & 138117005 & -5.5787 & -5.6172 \\
\hline SPNS1 & Spinster homolog 1 (Drosophila) & NM_032038 & chr 16 & & -5.9223 & -5.233 \\
\hline C6orf203 & Chromosome 6 open reading frame 203 & NM_016487 & chr 6 & 107456109 & -7.6213 & -3.4953 \\
\hline KIF18B & Kinesin family member $18 \mathrm{~B}$ & NM 001080443 & chr 17 & 40380608 & -6.0268 & -5.0785 \\
\hline C19orf50 & romosome 19 open reading frame 50 & NM_024069 & chr 19 & 18529603 & -7.6213 & -3.4712 \\
\hline TMEM219 & Transmembrane protein 219 & NM_001083613 & chr 16 & 29880851 & -6.9625 & -4.1224 \\
\hline SLC29A2 & family 29 (nucleoside transporters), me & NM_001532 & chr 11 & 65895867 & -6.3653 & -4.5099 \\
\hline ENG & & & & 129656805 & -7.6213 & -3.2082 \\
\hline MDM4 & Mdm4 p53 binding protein homolog (mouse) & NM_002393 & chr 1 & 202752133 & -7.6213 & -3.1811 \\
\hline
\end{tabular}

versus 1254 genes). The genes with both PcG marking and retrotransposon depletion (methylation-prone) had the highest levels of measured methylation, confirming that the two models complement each other.

\section{Refinement of the model}

Our data suggest that retrotransposon marking is a powerful discriminator of $\mathrm{CpG}$ island methylation predisposition in cancer, and that it compares favorably to models based on hyperconservation and PcG marking. However, it does not explain the be- havior of every single $\mathrm{CpG}$ island. Thus, there is room for refinement of the model, and combination of retrotransposon distribution with other features will likely improve its sensitivity and specificity. An important feature reported to predict methylation propensity of CpG islands are short DNA motifs discovered by Feltus et al. (2003, 2006), and recently modeled in a classifier called PatMan by McCabe et al. (2009). Applied to the first testing set, composed of genes known to be methylated according to the literature, PatMan identifies 9/142 genes as methylation-prone, and five of these (56\%) are indeed known to be methylated. Among the remaining 133 genes classified as methylation-resistant by PatMan, 
A.
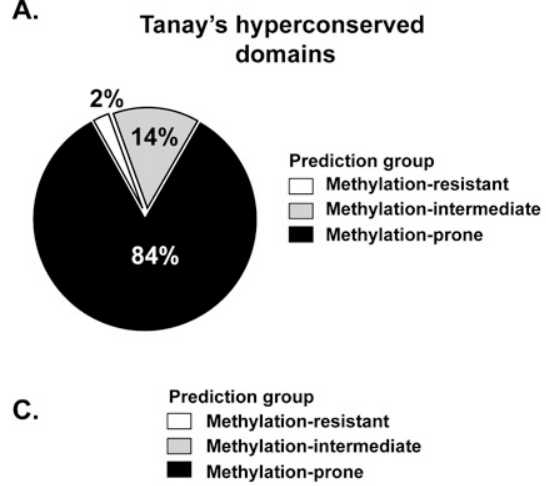

E.

\section{Validated hypermethylated} genes $(n=110)$

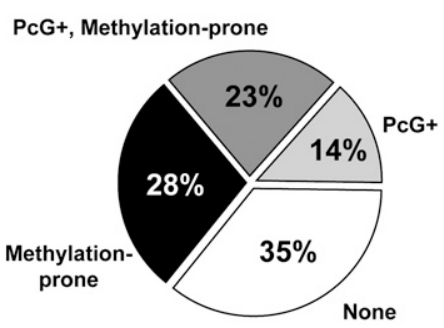

F.

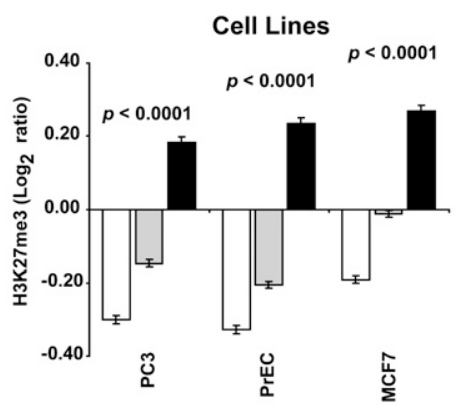

B.

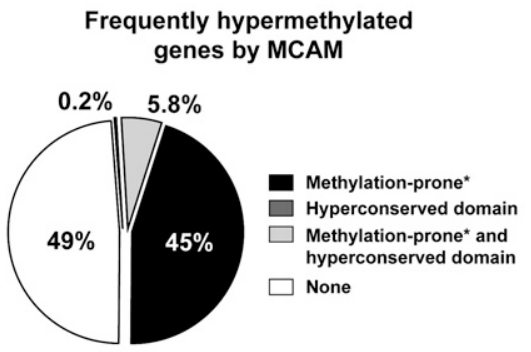

D.

ES Cells
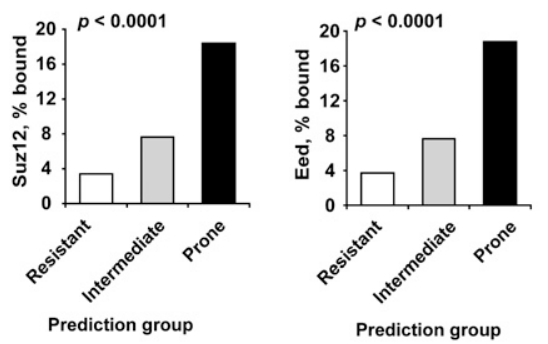

PcG marking, prediction group

I- PcG-, Methylation-Resistant III- PcG+, Methylation-resistant II- PcG-, Methylation-Prone IV- PcG+, Methylation-Prone

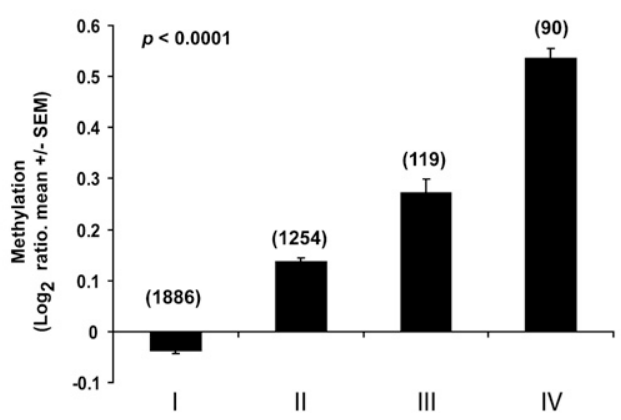

Figure 6. Genome architecture influences on PcG protein binding in embryonic and differentiated cells. (A) Frequency of predicted methylation groups among hyperconserved domains. (B) Relative contribution of hyperconserved domains and retrotransposon depletion in marking frequently methylated genes in cancer. MCAM data from 32 primary tissues and 28 cancer cell lines were averaged to identify frequently methylated genes. *Predicted status. (C) Enrichment of H3K27me3 mark in predicted methylation-prone genes in cancer (PC3, prostate; MCF7, breast) and normal immortalized (PrEC, prostate epithelium) cell lines. H3K27me3 marking was measured by ChIP with microarray hybridization (ChIP-chip) and is quantified as $\log _{2}$ ratio of pull-down signal over no antibody signal (Kondo et al. 2008). (D) Frequency of binding of SUZ1 2 and EED (PCG proteins) in human embryonic stem cells to 2583 methylation-resistant, 3655 methylation-intermediate, and 1690 methylation-prone genes based on our predictive model. Note that genes predicted methylation-prone (thus depleted for SINE and LINE retrotransposons) are preferential targets of PcG proteins. (E) Comparison of PcG marking and our predictive model in identifying methylation-prone genes from our training and first testing set. $(F)$ Average measured methylation of predicted methylation-prone and methylation-resistant genes in PCG marked genes. MCAM data from 32 primary tumors and 28 cancer cell lines were averaged per comparison group, and methylation is presented as $\log _{2}$ ratio (cancer/control). The number of genes per category is presented above each column.

69 are methylated. In comparison, 47/142 genes are classified as methylation-prone according to retrotransposon marking, and $38(81 \%)$ of these are indeed methylated. Thus, retrotransposon marking compares favorably to PatMan. In our validation using
MCAM data, PatMan is a good classifier of methylation-prone genes (Supplemental Fig. S7A), but it seems to lack the sensitivity of retrotransposon marking, as it misses a little more than one thousand methylation-prone CpG islands (Fig. 7A). PatMan does, however, resolve nearly 200 methylation-intermediate genes into the methylation-prone group.

Known insulator elements are likely to play a role in protection of $\mathrm{CpG}$ islands from de novo DNA methylation, especially if heterochromatin spreading, as suggested by several authors, is the main event leading to gene inactivation in cancer. To answer whether putative blocking elements influence gene promoter predisposition to DNA methylation, we compared the average methylation of genes with and without CTCF binding sites in the promoter vicinity. A gene promoter was considered to be bound by CTCF if this protein was present in the 2 -kb window centered on the gene TSS in at least one out of five normal adult cell lines investigated by chromatin immunoprecipitation with massively parallel sequencing (ChIP-seq) (Bernstein et al. 2005, 2006; Mikkelsen et al. 2007). According to this analysis, $\sim 36 \%$ of promoter $\mathrm{CpG}$ islands are bound by CTCF. Promoter CpG islands bound by CTCF seem to be protected from de novo methylation in cancer, as they present lower measured DNA methylation by MCAM compared to genes without CTCF (Fig. 7B). However, CTCF binding is not as strong a predictor of methylation predisposition as retrotransposons marking, since a large fraction of CTCF bound promoters do become methylated in cancer. When added to the prediction model, the presence of CTCF binding does not improve the classification of methylationresistant genes; even in the absence of CTCF, retrotransposons mark genes that do not become methylated in cancer (Supplemental Fig. S7B). However, the presence of CTCF decreases the methylation propensity of retrotransposon-poor promoters.

Finally, the breakdown of SINE and LINE retrotransposons in families and subfamilies may reveal additional information regarding their relationship to CpG island methylation. To test this possibility, we further annotated the presence of Alu and MIR repeats, the two main families of human SINE, in the $20-\mathrm{kb}$ window centered in the TSS of methylation-prone and methylation-resistant genes. This comparison revealed that $A l u$ repeats are the main drivers of the difference in abundance of SINE repeats between methylation-prone and methylation-resistant genes (Fig. 7C). The difference in frequency 
A.

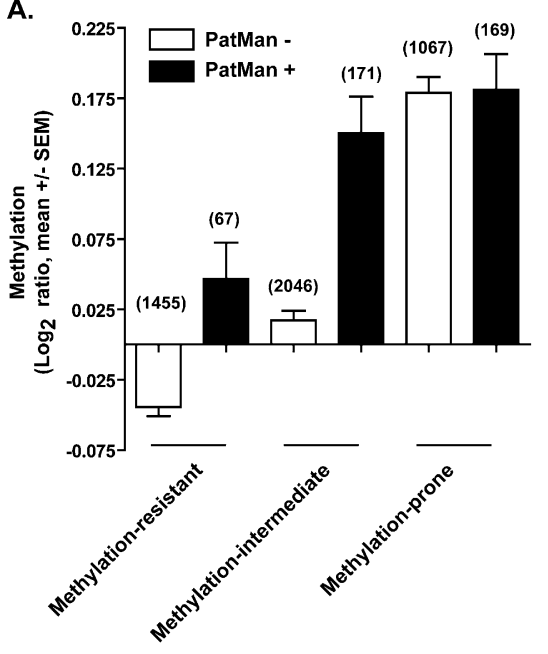

C.

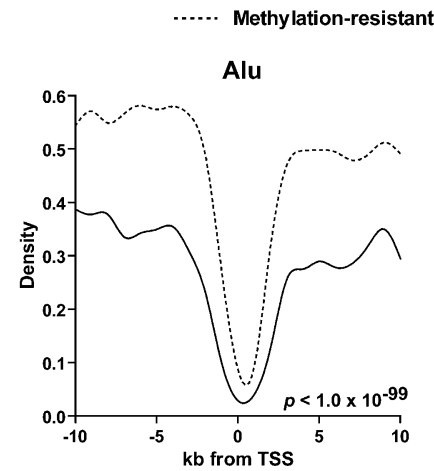

B.
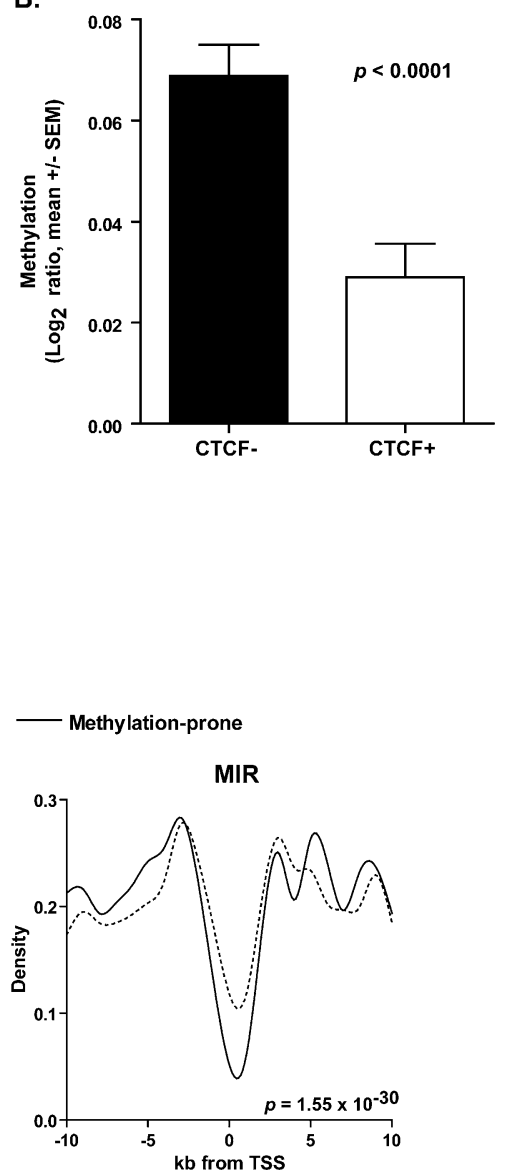

Figure 7. Other genomic features associated with methylation predisposition in cancer. $(A)$ Performance of PatMan and retrotransposon marking in predicting promoter $\mathrm{CpG}$ methylation status in cancer. PatMan-, predicted methylation-resistant genes; PatMan+, predicted methylation-prone genes. The number of genes per category is presented above each column. (B) Measured promoter $\mathrm{CpG}$ island methylation by MCAM of CTCF bound (CTCF+) and unbound (CTCF-) genes. A gene was considered bound by CTCF if ChIP-seq data from public databases show binding of this protein in the 2-kb region centered in the gene TSS. (C) Abundance of Alu and MIR retrotransposons in the 20-kb sequence window centered in the TSS of 740 methylation-prone and 5658 methylation-resistant genes. Note that the depletion of MIR retrotransposons is more significant in the $-2-k b$ to $+2-k b$ sequence window.

of MIR repeats is less evident, but these repeats are, like LINE repeats, depleted in the proximal region to TSS $(-2 \mathrm{~kb}$ to $+4 \mathrm{~kb})$.

\section{Discussion}

Our data show that a genome architecture marked by depletion of retrotransposons is strongly correlated to gene predisposition to DNA methylation in cancer. Moreover, we show that this same architecture is an independent predictor of gene expression in cancer and that it is correlated with PcG binding in embryonic stem cells and adult tissues. These data add to the list of possible influences of retrotransposons on genome biology. For example, SINEs are differentially distributed in imprinted (Greally 2002), tissue-specific (Ganapathi et al. 2005), and monoallelically expressed genes (Allen et al. 2003). Obviously, there may be overlap between these states. Indeed, in our study, the frequency of predicted methylation-prone and methylation-resistant genes among imprinted genes was significantly different from the genome-wide distribution of these methylation predisposition groups. These results were in agreement with previous studies showing that SINE repeats are less represented in imprinted genes, resulting in an underrepresentation of predicted methylation-resistant genes (Greally 2002). Of note, several imprinted genes predicted methylationprone have been reported as hypermethylated in cancer (for example, CDKN1C and $D L K 1)$. In contrast, despite reports of differential distribution of SINE and LINE retrotransposons on the $\mathrm{X}$ chromosome (Jurka et al. 2004; Wang et al. 2006), we did not find the frequency of methylation-prone and methylationresistant genes in the $\mathrm{X}$ chromosome to differ from autosomes. These results are consistent with the proposed idea that, if retrotransposons in fact participate in $\mathrm{X}$ inactivation, their action is due to longrange interactions rather than local, promoter-associated effects. It is important to note that all these studies are correlative, although they strongly point toward a role for retrotransposons in epigenetic regulation.

The depletion of SINE elements in methylation-prone genes is a paradoxical finding, since the main family of these elements (Alu family) is efficient as a methylation nucleation center in both plants and mammals (Yates et al. 1999; Arnaud et al. 2000), and the spreading of DNA methylation from these repeats into gene promoters was hypothesized to be a cause of de novo methylation in cancer (Jones and Baylin 2002). It is important to note, however, that an opposite effect has also been reported for repetitive elements, some of which have insulator function (Gdula et al. 1996; Lunyak et al. 2007). Although the exact mechanism by which the presence of retrotransposons is associated with protection from de novo promoter DNA methylation in cancer is unknown, it is possible that protection is not directly mediated by these repetitive elements but rather by transcription factors with euchromatin/heterochromatin boundary activity, such as CTCF (Bell et al. 1999) and Sp1 (Mummaneni et al. 1998) transcription factors. In such cases, genes without binding sites for boundary proteins would be negatively affected by the insertion of repetitive elements near their promoters. Thus, the presence of retrotransposons near these genes would be counterselected during evolution. Indeed, it has been shown in Arabidopsis thaliana that methylated transposable elements are preferentially inserted or retained in gene-poor areas, a feature that likely evolved due to negative selection (Hollister and Gaut 2009). Also, in agreement with this possibility, we show here that CTCF binding confers some protection from de novo methylation. We believe that the performance of CTCF binding alone as a predictor of methylation fate of gene promoters is somewhat weak due to two main factors: (1) CTCF binding should be taken in tissue-specific context, and compared to methylation data on the same tissue type; and (2) other insulator proteins, for which genome-wide binding to gene promoters has not yet been extensively 
reported (for example, VEZF1), should be taken into consideration together with CTCF when developing models of protection.

The lack of correlation of LINE repeats with methylation predisposition in mouse data sets is a puzzling observation, and a possible explanation resides in the fact that different repeats may impact the genome differently. Indeed, while SINE and LINE repeats are correlated to methylation predispositon, DNA repeats are not. Among SINE repeats, Alus showed higher correlation to promoter methylation status than MIRs. While not identical, human $A l u$ and mouse B1 share higher sequence similarity than human and mouse LINEs. Also, LINE-1 repeats, the main LINE family, are more variable in mice than in humans. In a direct comparison of repetitive elements in human and mouse genomes, a strong correlation was observed between the presences of SINE repeats in orthologous locations, an event quite surprising given the lineage specificity of these repeats (Mouse Genome Sequencing Consortium 2002). LINE repeats, however, do not show such correlation. Although these observations do not completely explain why mouse LINEs are not correlated to methylation predisposition, they point out that genomic location of different repeats may be dictated by the repeat structure itself and species-specific factors.

The fact that the frequency of retrotransposons alone can predict the expression status of thousands of genes in cancer supports the idea that epigenetic effects in cancer occur through an instructive mechanism and depend relatively little on gene function, at least initially. Indeed, the higher average methylation in PcG marked genes is due to concordant methylation status across different cell lines and primary tissues studied by MCAM, indicating that these genes are under a strong program to become epigenetically silenced. Retrotransposon-depleted genes are more heterogeneously methylated; their lack of protection from de novo methylation leaves these genes prone to epigenetic silencing, although it is not a programmed event and may depend on selection. Thus, as for genetic alterations, many of the methylation events in cancer are likely to be passengers, not drivers, in carcinogenesis. This does not exclude the active participation of a subset of methylation-prone genes with strong tumor-suppression function as drivers in tumorigenesis. Therefore, epigenetic defects in cancer are best viewed as a form of molecular instability creating diversity in gene expression that is exploited by cancers using classical selection mechanisms.

A possible explanation for the correlation between depletion of retrotransposons and predisposition to DNA methylation is that, similarly to PcG marking, retrotransposon depletion marks regulatory regions of developmental genes, which then might be especially prone to DNA methylation. Indeed, developmental genes often have hyperconserved CpG sites (Tanay et al. 2007). Most genes that have such hyperconservation were found to be predicted methylation-prone by our model, reinforcing the argument that retrotransposon depletion marks important essential genes for development. However, our results in primary bladder cancer methylation makes evident that tissue-lineage methylation is not a confounder in our results. In this experiment, bladder tissues from normal adult individuals were used as a control, thus eliminating tissue-specific methylation as a confounder. As shown, our predictive model performs well in this system after tissuespecific methylation has been removed. Still, these data do not exclude the possibility that chromatin states in normal tissues may influence DNA methylation. Indeed, as discussed above, this has been shown for PcG protein binding. It may be that our predicted methylation prone genes have a unique chromatin pattern in normal cells, beyond PcG protein binding, and this should be addressed in further studies. Independent of the mechanism by which retrotransposons are associated to or participate in protection from de novo DNA methylation in cancer, our findings suggest that the architecture of the genome has much more profound influences on cancer physiology than previously suspected.

\section{Methods}

\section{Cell lines and primary cancer samples}

A total of 34 cell lines from different tissues were obtained from the NCI Anticancer Drug Screen Panel, the American Type Culture Collection (Manassas, VA) or the University of Texas MD Anderson repositories (one cell line from breast cancer, 12 from bladder cancer, one from the central nervous system, four from colon cancer, 11 from leukemia, one from melanoma, one from lung cancer, one from ovarian cancer, one from prostate cancer, and one from renal cancer) and cultured according to standard methods.

Primary tumor samples from 28 bladder cancer and four acute myelogenous leukemia patients were collected at the MD Anderson Cancer Center in accordance with institutional policies. All patients provided written informed consent. Tumors were selected solely on the basis of availability. Genomic DNA was extracted from all cell lines and primary tissue samples using a standard phenol-chloroform method.

\section{Methylation analyses}

DNA methylation status of gene promoter CpG islands was evaluated using bisulfite-treated DNA followed by PCR amplification, and methylation density of individual genes was determined by pyrosequencing or COBRA assays according to standard protocols (Estecio et al. 2006, 2007). PCR primers are presented in Supplemental Tables S7 and S8. Genes with at least 15\% methylation density were considered hypermethylated (a conservative value chosen based on the detection limits of the methods) and genes hypermethylated in 2/9 tested cancer cell lines were classified as methylation-prone, given that in our research we intended to find genome signatures associated with predisposition or resistance to methylation across tissue types, rather than tissue-specific methylation. It is important to note, however, that the vast majority of the genes classified as methylation-prone showed gene methylation of $30 \%$ and higher, and in general they were methylated in three or more cell lines. Analysis of DNA methylation using the MCAM method was performed as previously reported (Estecio et al. 2007; Shen et al. 2007b). Tumor and normal control MCA amplicons were Cy5- and Cy3-labeled, respectively, and cohybridized to the HCGI12K-Human CpG 12K Array (Microarray Center, University Health Network, Toronto, Canada) or to a custom $4 \times$ $44 \mathrm{k}$ oligo array (Agilent Technologies). Methylation analysis of promoter $\mathrm{CpG}$ island methylation in mice was done using bone marrow samples of three NUP98-HOXD13 transgene animals that developed MDS (Lin et al. 2005). Bone marrow samples from a nontransgene animal of the same mouse strain were used as control in MCAM experiments, and the methylation status of approximately $6000 \mathrm{CpG}$ island promoter genes was determined using Mouse CpG Island Microarray Kit oligo arrays (Agilent Technologies). Additional data sets of promoter CpG island methylation in mouse tumors were obtained from published reports on a mouse model of chronic lymphocytic leukemia (Chen et al. 2009) and intestinal cancer (Hahn et al. 2008). Small intestine tissues isolated from young (3-mo-old) and old (35-mo-old) C57BL/6J mice and tumor samples from a leukemia mouse model (Choi et al. 2008) were used in MCAM experiments to identify cancer and age-related methylation. 


\section{Repetitive element abundance and CpG island characteristics}

The DNA sequence flanking the 4-kb sequence region surrounding the TSS of 36 methylation-prone and 36 methylation-resistant genes with a promoter CpG island was obtained from the University of California Santa Cruz (UCSC) BLAT Genome Browser (http://genome.ucsc.edu/cgi-bin/hgBlat). The TSS for each gene was determined according to RefSeq (NCBI Build 36.1), and repetitive DNA sequences were annotated using the RepeatMasker search engine (http://www.repeatmasker.org). All genome tables were downloaded from the UCSC Table Browser (http://genome. ucsc.edu/cgi-bin/hgTables; Karolchik et al. 2004). For all statistical analysis, CpG island length, GC content, and CpG ratio were calculated using the public software CpG Island Searcher (http:// www.cpgislands.com; Takai and Jones 2003).

\section{Calculation of a score to predict gene promoter predisposition to DNA methylation}

We divided the promoter sequence of 36 methylation-prone and 36 methylation-resistant genes into 20 bins of 1-kb sequence each (10 bins upstream and 10 bins downstream of each gene TSS), and annotated the presence SINE and LINE retrotransposons in each bin (Fig. 2A). In order to avoid a single repeat being counted in multiple bins, SINE and LINE elements were annotated to the closest bin to TSS. Done this way, each gene is represented by a 20-letter acronym, where $S$ represents a SINE element in the bin and $\mathrm{G}$ represents the absence of a SINE element (Fig. 2B). Similarly, an independent acronym is generated for LINE repeats, where $L$ represents a LINE element in the bin and G represents the absence of a LINE element. We compared the average abundance of SINE and LINE retrotransposons per bin in methylation-prone and methylation-resistant genes to their average abundance genome-wide in the full collection of human promoter CpG islands, and translated the preference to repetitive elements to a score that differentiates two kinds of promoters.

Such a score is a sum over the bin score, which is a standard log-odds ratio:

$$
s_{i, r}=\ln \left(\frac{q_{i, r}}{p_{r}}\right)
$$

where $q_{i, r}$ is the frequency of observing repeat of type $r$ for the $i$ th bin for the promoters known to be methylated; $p_{r}$ is the background frequency for the repeat $r$. To account for the low count and avoid taking logarithm of zero, $q_{i, r}$ is replaced by $Q_{i, r}=\frac{c_{i, r}+f_{r}}{N+1}$ derived from "pseudo-count," where $f_{r}$ is the fraction of the repeat that is type $r: \sum_{r} f_{r}=1 . N$ is the total number of promoters with the known methylation status; $c_{i, r}$ is the number of repeats of type $r$ in the $i$ th bin: $\sum_{r} c_{i, r}=N$. The final value for each letter in the 20-letter acronym representing the abundance of SINE and LINE elements was calculated as the difference between its value in methylation-prone and methylation-resistant genes (for example, $s_{10, S}=s_{10, S m p}-s_{10, S m r}$, where $S m p$ is the SINE standard log-odds ratio in methylation-prone genes and Smr is the SINE standard logodds ratio in methylation-resistant genes). The calculation of the log-odds ratios for SINE elements is illustrated in Figure 2C.

\section{Identification of clusters of methylation-prone genes}

To identify clusters of methylation-prone genes, we computed the correlation as a function of separation between genes. We denoted the computed status of whether the ith gene on a chromosome is methylation-prone by $S_{i}$, where $S_{i}=1$ indicates methylation-prone and $S_{i}=0$ indicates methylation-resistance. The following correlation function was applied to detect clustering of methylationprone or methylation-resistance: $C(d)$ where $L$ is the total number of genes in the chromosome. We similarly defined the correlation function over the genome by averaging over all chromosomes. At a large gene separation (distance $d$ ), $S_{i}$ and $S_{i+d}$ become independent. If there is a correlation at a short distance, it will result in an elevated value of $C(d)$ at a shorter distance in comparison to a larger distance. We found that between distances 3 and 60 , the correlation $C(d)$ can be fitted to an exponential function $e^{\frac{d}{-l}}$ where $l=20.6$ indicates a decay length of about 21 gene separations. In these analyses, only genes with promoter CpG islands were computed. In order to find the regions of clustering of methylationprone genes, we look for a string of consecutive methylation-prone genes $(i=1)$ in $S_{i}$. We compared the length of the string with what is allowed by random chance. Let $N_{0}$ and $N_{1}$ be the number of 0 's (methylation-resistant) and 1's in $S_{i}$, then $p_{1}=\frac{N_{1}}{N_{0}+N_{1}}$ and $p_{0}=1-p_{1}$. If 0 's and 1's are randomly distributed, the chance of having $L-m 1$ 's and $m 0$ 's is given by $P(L)=\left(\begin{array}{l}L \\ m\end{array}\right) p_{1}^{L-m} p_{0}^{m}$. Let $N=N_{0}=N_{1}$, using very conservative Bonferroni correction for multiple testings, when $P(L) \times N \approx 1$, there is approximately one string with $L-m 1$ 's and 0 's in the entire genome. To control for multiple $L$ we find the smallest $L$ that satisfies $N \times \sum_{1=L}^{N} P(L) \leq 1$. Although $P / L$ over $L$ does not sum to one, we are summing over very small $P / L$ as an approximation to control for multiple string length tested. So for $m=0$ we find all string of 1's with length $\geq L$ (the threshold value in this case is $L=7$ ) and mark this region as significant. Similarly, we mark regions for $m$ up to 10 .

\section{Gene expression, ChIP-chip, and PatMan data sets}

Gene expression profiles of normal tissues were downloaded from GNF expression database (http://expression.gnf.org/; Su et al. 2004). Raw expression values in each data set were substituted by their respective $Z$-scores ( $[X-\mu] / \sigma$, where $X$ represents expression data of each gene in each sample; $\mu$ represents mean of expression of all genes for each sample; and $\sigma$ represents standard deviation). $S$ and $L$ scores were attributed to each gene, and $Z$-score expression data were averaged per predicted methylation status (prone or resistant to methylation) according to tissue of origin. Gene expression profiles of cancer cell lines were downloaded from the NCI60 Cancer Microarray Project website (http://genome-www. stanford.edu/nci60/; Ross et al. 2000) and analyzed as described for normal tissue expression data. Suz12 and Eed polycomb group (PcG) protein binding and H3K27me3 ChIP data are from human embryonic stem cells (Lee et al. 2006), and from PC3 (prostate cancer cell line), MCF7 (breast cancer cell line), and PrEC (immortalized normal prostate epithelial cells) (Kondo et al. 2008). CTCF binding data are from normal cultured cells (Bernstein et al. 2005, 2006; Mikkelsen et al. 2007). Genome-wide classification of CpG island in methylation-prone and methylation-resistant genes according to the PatMan classifier was retrieved from McCabe et al. (2009).

\section{Gene Ontology analysis}

We used FatiGO (Al-Shahrour et al. 2006) from Babelomics (http:// babelomics.bioinfo.cipf.es/) for Gene Ontology analysis. The statistical significance of frequency of genes per biological process in predicted methylation-prone and methylation-resistant groups was calculated using Fisher's exact test. $P$-values were adjusted for false discovery rate.

\section{Acknowledgments}

This work was supported by the Leukemia Specialized Program of Research Excellence grant P50 CA100632, the National Institutes of Health grants R01 CA098006 and U01 CA085078, and the NIH intramural research program. J.P.J.I. is an American Cancer Society 
Clinical Research Professor. We thank Stephanie P. Deming for editorial help.

\section{References}

Allen E, Horvath S, Tong F, Kraft P, Spiteri E, Riggs AD, Marahrens Y. 2003. High concentrations of long interspersed nuclear element sequence distinguish monoallelically expressed genes. Proc Natl Acad Sci 100: 9940-9945.

Al-Shahrour F, Minguez P, Tarraga J, Montaner D, Alloza E, Vaquerizas JM, Conde L, Blaschke C, Vera J, Dopazo J. 2006. BABELOMICS: A systems biology perspective in the functional annotation of genome-scale experiments. Nucleic Acids Res 34: W472-W476.

Arnaud P, Goubely C, Pelissier T, Deragon JM. 2000. SINE retroposons can be used in vivo as nucleation centers for de novo methylation. Mol Cell Biol 20: $3434-3441$

Baylin S, Bestor TH. 2002. Altered methylation patterns in cancer cell genomes: Cause or consequence? Cancer Cell 1: 299-305.

Bell AC, West AG, Felsenfeld G. 1999. The protein CTCF is required for the enhancer blocking activity of vertebrate insulators. Cell 98: 387-396.

Bernstein BE, Kamal M, Lindblad-Toh K, Bekiranov S, Bailey DK, Huebert DJ, McMahon S, Karlsson EK, Kulbokas EJ III, Gingeras TR, et al. 2005 Genomic maps and comparative analysis of histone modifications in human and mouse. Cell 120: $169-181$

Bernstein BE, Mikkelsen TS, Xie X, Kamal M, Huebert DJ, Cuff J, Fry B, Meissner A, Wernig M, Plath K, et al. 2006. A bivalent chromatin structure marks key developmental genes in embryonic stem cells. Cell 125: $315-326$.

Bock C, Paulsen M, Tierling S, Mikeska T, Lengauer T, Walter J. 2006. CpG island methylation in human lymphocytes is highly correlated with DNA sequence, repeats, and predicted DNA structure. PLoS Genet 2: e26. doi: 10.1371/journal.pgen.0020026.

Chen SS, Raval A, Johnson AJ, Hertlein E, Liu TH, Jin VX, Sherman MH, Liu SJ, Dawson DW, Williams KE, et al. 2009. Epigenetic changes during disease progression in a murine model of human chronic lymphocytic leukemia. Proc Natl Acad Sci 106: 13433-13438.

Choi CW, Chung YJ, Slape C, Aplan PD. 2008. Impaired differentiation and apoptosis of hematopoietic precursors in a mouse model of myelodysplastic syndrome. Haematologica 93: 1394-1397.

Das R, Dimitrova N, Xuan Z, Rollins RA, Haghighi F, Edwards JR, Ju J, Bestor TH, Zhang MQ. 2006. Computational prediction of methylation status in human genomic sequences. Proc Natl Acad Sci 103: 10713-10716.

Estecio MR, Youssef EM, Rahal P, Fukuyama EE, Gois-Filho JF, Maniglia JV, Goloni-Bertollo EM, Issa JP, Tajara EH. 2006. LHX6 is a sensitive methylation marker in head and neck carcinomas. Oncogene 25: 5018-5026.

Estecio MR, Yan PS, Ibrahim AE, Tellez CS, Shen L, Huang TH, Issa JP. 2007. High-throughput methylation profiling by MCA coupled to CpG island microarray. Genome Res 17: 1529-1536.

Faulkner GJ, Kimura Y, Daub CO, Wani S, Plessy C, Irvine KM, Schroder K, Cloonan N, Steptoe AL, Lassmann T, et al. 2009. The regulated retrotransposon transcriptome of mammalian cells. Nat Genet 41: 563-571.

Feltus FA, Lee EK, Costello JF, Plass C, Vertino PM. 2003. Predicting aberrant CpG island methylation. Proc Natl Acad Sci 100: 12253-12258.

Feltus FA, Lee EK, Costello JF, Plass C, Vertino PM. 2006. DNA motifs associated with aberrant CpG island methylation. Genomics 87: 572-579.

Ganapathi M, Srivastava P, Das Sutar SK, Kumar K, Dasgupta D, Pal Singh G, Brahmachari V, Brahmachari SK. 2005. Comparative analysis of chromatin landscape in regulatory regions of human housekeeping and tissue specific genes. BMC Bioinformatics 6: 126. doi: 10.1186/1471-2105-6-126.

Gardiner-Garden M, Frommer M. 1987. CpG islands in vertebrate genomes. $J$ Mol Biol 196: 261-282.

Gdula DA, Gerasimova TI, Corces VG. 1996. Genetic and molecular analysis of the gypsy chromatin insulator of Drosophila. Proc Natl Acad Sci 93: 9378-9383.

Greally JM. 2002. Short interspersed transposable elements (SINEs) are excluded from imprinted regions in the human genome. Proc Natl Acad Sci 99: 327-332.

Hahn MA, Hahn T, Lee DH, Esworthy RS, Kim BW, Riggs AD, Chu FF, Pfeifer GP. 2008. Methylation of polycomb target genes in intestinal cancer is mediated by inflammation. Cancer Res 68: 10280-10289.

Han JS, Szak ST, Boeke JD. 2004. Transcriptional disruption by the L1 retrotransposon and implications for mammalian transcriptomes. Nature 429: 268-274.

Hollister JD, Gaut BS. 2009. Epigenetic silencing of transposable elements: A trade-off between reduced transposition and deleterious effects on neighboring gene expression. Genome Res 19: 1419-1428.

Jones PA, Baylin SB. 2002. The fundamental role of epigenetic events in cancer. Natl Rev 3: 415-428.

Jurka J, Kohany O, Pavlicek A, Kapitonov VV, Jurka MV. 2004. Duplication, coclustering, and selection of human Alu retrotransposons. Proc Natl Acad Sci 101: 1268-1272.
Karolchik D, Hinrichs AS, Furey TS, Roskin KM, Sugnet CW, Haussler D, Kent WJ. 2004. The UCSC Table Browser data retrieval tool. Nucleic Acids Res 32: D493-D496.

Kondo E, Furukawa T, Yoshinaga K, Kijima H, Semba S, Yatsuoka T, Yokoyama T, Fukushige S, Horii A. 2000. Not hMSH2 but hMLH1 is frequently silenced by hypermethylation in endometrial cancer but rarely silenced in pancreatic cancer with microsatellite instability. Int J Oncol 17: 535-541.

Kondo Y, Shen L, Cheng AS, Ahmed S, Boumber Y, Charo C, Yamochi T, Urano T, Furukawa K, Kwabi-Addo B, et al. 2008. Gene silencing in cancer by histone $\mathrm{H} 3$ lysine 27 trimethylation independent of promoter DNA methylation. Nat Genet 40: 741-750.

Lee TI, Jenner RG, Boyer LA, Guenther MG, Levine SS, Kumar RM, Chevalier B, Johnstone SE, Cole MF, Isono K, et al. 2006. Control of developmental regulators by Polycomb in human embryonic stem cells. Cell 125: 301-313.

Lin YW, Slape C, Zhang Z, Aplan PD. 2005. NUP98-HOXD13 transgenic mice develop a highly penetrant, severe myelodysplastic syndrome that progresses to acute leukemia. Blood 106: 287-295.

Lunyak VV, Prefontaine GG, Núñez E, Cramer T, Ju BG, Ohgi KA, Hutt K, Roy R, García-Díaz A, Zhu X, et al. 2007. Developmentally regulated activation of a SINE B2 repeat as a domain boundary in organogenesis. Science 317: 248-251.

McCabe MT, Lee EK, Vertino PM. 2009. A multifactorial signature of DNA sequence and polycomb binding predicts aberrant $\mathrm{CpG}$ island methylation. Cancer Res 69: 282-291.

Mikkelsen TS, Ku M, Jaffe DB, Issac B, Lieberman E, Giannoukos G, Alvarez P, Brockman W, Kim TK, Koche RP, et al. 2007. Genome-wide maps of chromatin state in pluripotent and lineage-committed cells. Nature 448: $553-560$.

Mouse Genome Sequencing Consortium. 2002. Initial sequencing and comparative analysis of the mouse genome. Nature 420: $520-562$.

Mummaneni P, Yates P, Simpson J, Rose J, Turker MS. 1998. The primary function of a redundant Sp1 binding site in the mouse aprt gene promoter is to block epigenetic gene inactivation. Nucleic Acids Res 26: 5163-5169.

Ohm JE, McGarvey KM, Yu X, Cheng L, Schuebel KE, Cope L, Mohammad HP, Chen W, Daniel VC, Yu W, et al. 2007. A stem cell-like chromatin pattern may predispose tumor suppressor genes to DNA hypermethylation and heritable silencing. Nat Genet 39: 237-242.

Ross DT, Scherf U, Eisen MB, Perou CM, Rees C, Spellman P, Iyer V, Jeffrey SS, Van de Rijn M, Waltham M, et al. 2000. Systematic variation in gene expression patterns in human cancer cell lines. Nat Genet 24: 227-235.

Schlesinger Y, Straussman R, Keshet I, Farkash S, Hecht M, Zimmerman J, Eden E, Yakhini Z, Ben-Shushan E, Reubinoff BE, et al. 2007. Polycombmediated methylation on Lys27 of histone H3 pre-marks genes for de novo methylation in cancer. Nat Genet 39: 232-236.

Shen L, Kondo Y, Ahmed S, Boumber Y, Konishi K, Guo Y, Chen X, Vilaythong JN, Issa JP. 2007a. Drug sensitivity prediction by CpG island methylation profile in the NCI-60 cancer cell line panel. Cancer Res 67: 11335-11343.

Shen L, Kondo Y, Guo Y, Zhang J, Zhang L, Ahmed S, Shu J, Chen X, Waterland RA, Issa JP. 2007b. Genome-wide profiling of DNA methylation reveals a class of normally methylated CpG island promoters. PLoS Genet 3: e181. doi: 10.1371/journal.pgen.0030181.

Simons C, Pheasant M, Makunin IV, Mattick JS. 2006. Transposon-free regions in mammalian genomes. Genome Res 16: 164-172.

Su AI, Wiltshire T, Batalov S, Lapp H, Ching KA, Block D, Zhang J, Soden R, Hayakawa M, Kreiman G, et al. 2004. A gene atlas of the mouse and human protein-encoding transcriptomes. Proc Natl Acad Sci 101: 6062-6067.

Takai D, Jones PA. 2003. The CpG island searcher: A new WWW resource. In Silico Biol 3: 235-240.

Tanay A, O'Donnell AH, Damelin M, Bestor TH. 2007. Hyperconserved CpG domains underlie Polycomb-binding sites. Proc Natl Acad Sci 104: 55215526.

Toyota M, Issa JP. 1999. CpG island methylator phenotypes in aging and cancer. Semin Cancer Biol 9: 349-357.

Wang Z, Willard HF, Mukherjee S, Furey TS. 2006. Evidence of influence of genomic DNA sequence on human $\mathrm{X}$ chromosome inactivation. PLoS Comput Biol 2: e113. doi: 10.1371/journal.pcbi.0020113.

Weber M, Hellmann I, Stadler MB, Ramos L, Paabo S, Rebhan M, Schubeler D. 2007. Distribution, silencing potential and evolutionary impact of promoter DNA methylation in the human genome. Nat Genet 39: 457-466.

Widschwendter M, Fiegl H, Egle D, Mueller-Holzner E, Spizzo G, Marth C, Weisenberger DJ, Campan M, Young J, Jacobs I, et al. 2007. Epigenetic stem cell signature in cancer. Nat Genet 39: 157-158.

Yates PA, Burman RW, Mummaneni P, Krussel S, Turker MS. 1999. Tandem B1 elements located in a mouse methylation center provide a target for de novo DNA methylation. J Biol Chem 274: 36357-36361.

Yoder JA, Walsh CP, Bestor TH. 1997. Cytosine methylation and the ecology of intragenomic parasites. Trends Genet 13: 335-340.

Received March 4, 2010; accepted in revised form July 15, 2010.

\section{Genome Research}

www.genome.org 


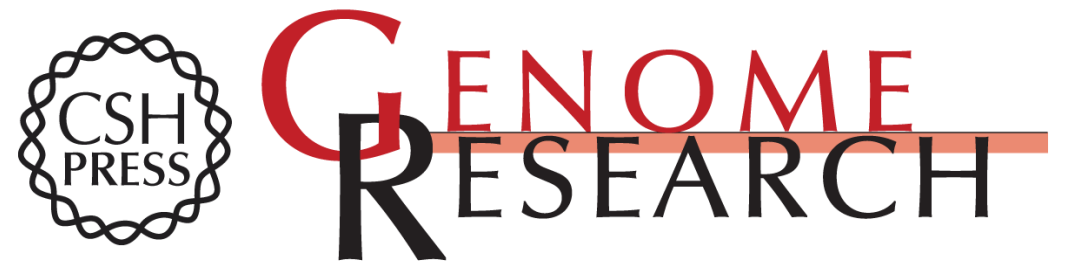

\section{Genome architecture marked by retrotransposons modulates predisposition to DNA methylation in cancer}

Marcos R.H. Estécio, Juan Gallegos, Céline Vallot, et al.

Genome Res. 2010 20: 1369-1382 originally published online August 17, 2010

Access the most recent version at doi:10.1101/gr.107318.110

Supplemental Material

References

License

Email Alerting Service
http://genome.cshlp.org/content/suppl/2010/08/18/gr.107318.110.DC1

This article cites 50 articles, 20 of which can be accessed free at: http://genome.cshlp.org/content/20/10/1369.full.html\#ref-list-1

Receive free email alerts when new articles cite this article - sign up in the box at the top right corner of the article or click here.

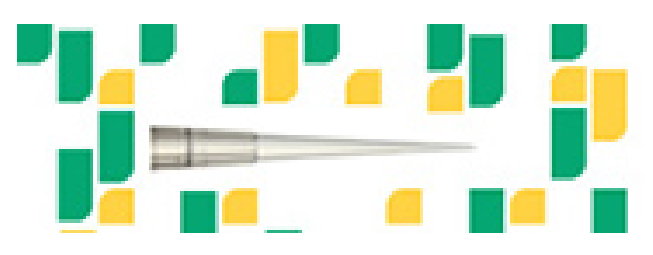

Focused on your science.

J⿹勹ి

SCIENTIFIC

suos or seisnes

To subscribe to Genome Research go to: https://genome.cshlp.org/subscriptions 